\title{
GENERAL INDEX TO VOLUME 68 (2011)
}

Note: Main entries are emboldened; * signifies the page range of keywords; pages with illustrations (Fig.), maps, appendices (App.) and reviews (Rev.) are indicated; tables are not annotated separately.

Aaronsohnia

factorovskyi Warb. \& Eig, 203, 205, 217 App.

Abelmoschus

esculentus (L.) Moench, 305, 312

manihot (L.) Medik., 309

Abies, 85, 97

Acacia, 187, 315, 388

ehrenbergiana Hayne, 187, 196 App.

gentlei Standl., 292 App.

gerrardii Benth.

var. najdensis Zohary, 202, 204-206, 221 App.

hamulosa Benth., 187, 196 App.

oerfota (Forssk.) Schweinf., 187-188, 196

App.

tortilis (Forssk.) Hayne, 187-190

[190 Fig.], 196 App.

Acanthaceae, 193 App., 214 App., 312, 333-337*, 425

subtribe Justiciinae, 333

Achariaceae, 325 Rev., 388

Achillea

fragrantissima (Forssk.) Sch.Bip., 217 App.

Achyranthes

aspera L., 309

Acoelorraphe

wrightii (Griseb. \& H.Wendl.) H.Wendl. ex Becc., 274

Aconitum, 158 Rev.

Acosmium

dasycarpum (Vogel) Yakovlev, 407

Additions, to flora of Gabon, 423-442

Adenium

obesum (Forssk.) Roem. \& Schult., 308

Adenocline Turcz., 444

Adiantaceae, 329 Rev.

Adiantum

capillus-veneris L., 205, 223 App.

lunulatum Burm.f., 308

Adinandra Jack, 41

Aegilops

kotschyi Boiss., 219 App.
Aerva

javanica (Burm.f.) Juss. ex Schult., 193

App., 214 App.

Aeschynomene uniflora E.Mey., 429

Aextoxicaceae, 157 Rev.

Aframomum angustifolium (Sonn.) K.Schum., 438

thonneri De Wild., 438

Africa

Central, 423-442*

crossroad with Asia, 301-319

South, 351-371

West, 343-350*

Agapetes D.Don, 422

Agarista

oleifolia (Cham.) G.Don, 386

Agathisanthemum, 424, 432

globosum (Hochst. ex A.Rich.) Klotzsch, 432

Agavaceae, 291 App.

Agave, 291 App.

Agouti

paca L., 287

Agrostistachys

staminodiatus Sevilla, 478

Aidia, 11

Aizoaceae, 214 App.

Aizoanthemum

hispanicum (L.) H.E.K.Hartmann, 214

App.

Aizoon

canariense L., 214 App.

hispanicum L., 214 App.

Al-AвbAsi, T.M., 183-197, 199-224

Alchornea

latifolia Sw., 292 App.

triplinervia (Spreng.) Müll.Arg., 386, 391

Al-Harbi, R.J., 183-197, 199-224

Alibertia, 406

edulis (Rich.) A.Rich. ex DC., 406-407

myrciifolia Spruce ex K.Schum., 407

sessilis (Vell.) K.Schum., 408 


\section{Alkanna}

orientalis (L.) Boiss., 215 App.

Alliaceae, 214 App.

Allium stramineum Boiss., 206, 214 App.

Allophylus rubifolius (A.Rich.) Engl. var. rubifolius, 306

Aloe praetermissa McCoy \& Lavranos, 309, 312

Al-Shammari, K.F., 183-197, 199-224

Al-Shenbaz, I.A., 165-171

Althaea ludwigii L., 221 App.

Alves, R.J.V., 33-38

Al-Wetaid, A.H., 199-224

Alysicarpus

glumaceus (Vahl) DC., 305, 312 vaginalis (L.) DC., 307

Alyssum homalocarpum, 215 App.

Amaioua guianensis Aubl., 412-413, 415

Amaranthaceae, 193 App., 214 App.

Amaranthus graecizans L., 215 App. viridis L., 215 App.

Amarasinghe, A.P.P.R., 333-337

Amborellaceae, 157 Rev.

America, Central, 273-296

Ammoperdix heyi Temminck, 190

Anabasis setifera Moq., 193 App.

Anacardiaceae, 215 App., 388, 391

Anadenanthera colubrina (Vell.) Brenan, 386, 413

Anagallis

arvensis L., 221 App., 307

var. caerulea (L.) Gouan, 221 App.

pumila $\mathrm{Sw} ., 309$

Anaimalais, India, 173-175*

Anatomy of Calolisianthus, 139-155* of Gagea, 43-59*

Anchusa aegyptiaca (L.) A.DC., 215 App. hispida Forssk., 194 App. milleri Spreng., 215 App.
Ancistrocladaceae, $329 \mathrm{Rev}$.

Andira

cuiabensis Benth., 412-413, 415

fraxinifolia Benth., 386, 391

Andrachne aspera Spreng., 206, 222 App., 309

telephioides L., 206, 222 App.

Anisoptera, 468

Anisosciadium lanatum Boiss., 206, 224 App.

Annesijoa, 469

Annona coriacea Mart., 408 crassiflora Mart., 408-409

Annonaceae, 173-175*, 291 App., 339-341, 387-388, 392

Anogeissus, 302, 313, 315

dhofarica A.J.Scott, 302, 306, 311-312

Antalya, Turkey, 159-163*

Anthemis, 202 melampodina Delile, 217 App. pseudocotula Boiss., 205, 217 App. zoharyana Eig, 217 App.

Antidesma leptobotryum Müll.Arg., 345

mannianum Müll.Arg., 348-349

stenopetalum Müll.Arg., 348-349

vogelianum Müll.Arg., 349

Antirrhinum orontium L., 222 App.

Antonia ovata Pohl, 386, 408

Anvillea garcinii (Burm.f.) DC., 203, 205, 217 App. Apiaceae, 224 App.

Apluda mutica L., 305, 311-312

Apocynaceae, 194 App., 215 App., 291 App., 392, 406, 426

Aporosa Blume, 478

Appendicula Blume, 321-324* cornuta Blume, 321

nicobarica Jayanthi, Sumathi \& Karthig., 321-324 [322 Fig.]

pendula Blume, 321, 323

reflexa Blume, 321

Aquifoliaceae, 291 App.

Arabia, southern, 301-319* [303 Map]

Arabian Peninsula, IPAs in, 183-197, 199-224 


\section{Arabidopsis}

erysimoides Hedge \& Kit Tan, 216 App. Araucaria, 468

Araucariaceae, 329 Rev.

ARAúJo, G.M., 401-418

Arbor regis Rumph., 470

ARDI, W.H., 225-255

Arecaceae, 279, 291 App., 387-388

Arenaria

foliacea Turrill, 205, 216 App.

Argent, G., 419-422

Argent, G.C.G., 39-42

Arias Guerrero, S., 443-482

Aristida adscensionis L., 187, 195 App., 219 App.

Arnebia decumbens (Vent.) Coss. \& Kralik, 215 App.

hispidissima (Sieber ex Lehm.) A.DC., 194 App., 215 App.

linearifolia A.DC., 215 App.

Artemisia

judaica L., 206, 217 App.

Arthraxon

micans (Nees) Hochst., 307

pusillus Bor, 305, 312

Arundinella

pumila (Hochst. ex A.Rich.) Steud., 309

Ascarina J.R.Forst. \& G.Forst., 41

Asclepias

kamerunensis Schltr., 425-426

Asia

crossroad with Africa, 301-319

South, 339-341

Asperugo

procumbens L., 215 App.

Asphodelaceae, 215 App., 312

Asphodelus tenuifolius Cav., 202-203, 205, 215 App.

Aspidosperma, 406

cuspa (Kunth) S.F.Blake ex Pittier, 409

dispermum Müll.Arg., 392

macrocarpon Mart., 407

tomentosum Mart., 407

Asteraceae, 194 App., 217 App., 281, 291

App., 297, 312, 392, 424, 427

Asteriscus

pygmaeus (DC.) Coss. \& Durieu, 205, 217

App.
Astragalus, 50

collenettiae Hedge \& Podl., 207, 209, 221

App.

crenatus Schult., 221 App.

hamosus L., 221 App.

hauarensis Boiss., 187, 196 App.

schimperi Boiss., 221 App.

sieberi DC., 203, 221 App.

spinosus (Forssk.) Muschl., 196 App., 203, 205, 221 App.

tribuloides Delile, 196 App., 221 App.

vogelii (Webb) Bornm., 196 App.

Astrocaryum aculeatissimum (Schott) Burret, 388

Astronium

fraxinifolium Schott ex Spreng., 407

Atherospermataceae, $157 \mathrm{Rev}$.

Atlantic Semideciduous Forests, 373-400*

Atractocarpus Schltr. \& K.Krause, 30 obscurinervius (Merr.) Puttock, 30

Atractylis

cancellata L., 217 App.

carduus (Forssk.) C.Chr., 194 App., 217

App.

flava Desf., 217 App.

Avena

barbata Pott ex Link, 205, 219 App.

sterilis L., 219 App.

Azavedo, A.A., 139-155

Baccaureopsis Pax, 344

Balanites

wilsonianus Dawe \& Sprague, 439

var. glabripetalus Sands, 438-439

var. mayumbensis (Exell) Sands, 439

var. wilsonianus, 439

Balanophoraceae, 329-330 Rev.

Ballota

undulata (Sieber ex Fresen.) Benth., 202, 205, 220 App.

Banara

kuhlmannii (Sleumer) Sleumer, 388

Baphia, 424

longepedicellata Willd.

subsp. longepedicellata, 429

Bauhinia, 408

Beddome, R.H., Icones Plantarum of, 173-175*

Begonia L., 177-182*, 225-255*

sect. Diploclinium (Lindl.) A.DC., 232, 243

sect. Eupetalum (Lindl.) A.DC., 177-178 
sect. Knesebeckia (Klotzsch) A.DC., 177, 180-181

sect. Petermannia (Klotzsch) A.DC., 225-226, 230, 232-233, 235, 237, 240, 243, 246, 248

sect. Sphenanthera (Hassk.) Warb., 243 aptera Blume, 225, 254

asympeltata L.B.Sm. \& Wassh., 177-178, 180-181

bifurcata L.B.Sm. \& B.G.Schub., 177, 180-181

bonthainensis Hemsl., 252

celebica Irmsch., 243, 253

comestibilis D.C.Thomas \& Ardi,

225-230 [226, 227 Map, 228 Fig.], 251

compacticaulis Irmsch., 177, 180-181

didyma D.C.Thomas \& Ardi, 233, 252

dioica Buch.-Ham. ex D.Don, 243

erythrocarpa A.DC., 180

gorgonea Tebbitt, 177-181 [178, 179 Fig.]

grandipetala Irmsch., 252

guttapila, 230, 243, 251, 253

imperfecta Irmsch., 243, 246, 248, 253

insueta D.C.Thomas \& Ardi, 225-227

[227 Map], 230-232 [231 Fig.], 251

lasioura D.C.Thomas \& Ardi, 225, 227

Map, 233-235 [234 Fig.], 253

lutea L.B.Sm. \& B.G.Schub., 177

mekonggensis Girm. \& Wiriad., 243, 252

nobmanniae D.C.Thomas \& Ardi, 225,

227 Map, 235-237 [236 Fig.], 253

octopetala, 178

oligandra Merr. \& L.M.Perry, 232

ozothrix D.C.Thomas, 252

parcifolia C.DC., 177, 180-181

polilloensis Tebbitt, 232

prionota D.C.Thomas \& Ardi, 225, 227

Map, 237-240 [238 Fig.], 253

rachmatii Tebbitt, 232, 235, 237, 251

rantemarioensis D.C.Thomas \& Ardi,

225, 227 Map, 240-243 [241 Fig.], 246,

248, 251, 253

rieckei Warb., 225, 230, 254

robusta Blume, 254

sanguineopilosa D.C.Thomas \& Ardi,

225, 227 Map, 230, 240, 243-246 [244

Fig.], 251, 253

sarasinorum Irmsch., 243, 252

serotina A.DC., 177-178, 180-181

siccacaudata J.Door., 251 sphenocarpa Irmsch., 243, 253-254

torajana D.C.Thomas \& Ardi, 225, 227

Map, 230, 243, 246-248 [247 Fig.], 251, 253

triramosa Irmsch., 177, 180-181

varipeltata D.C.Thomas, 230

vermeulenii D.C.Thomas, 225-227

[227 Map], 230, 248-251 [249 Fig.]

Begoniaceae, 177-182, 225-255

Belize, 273-296*

Belonophora coriacea, 433

ongensis S.E.Dawson \& Cheek, 433

Berberidopsidaceae, 157 Rev.

Berberidopsis, 158 Rev.

Berg, C.C., 269-272

Bertiera orthopetala (Hiern) N.Hallé, 433

Beta vulgaris L., 215 App.

Betula, 97

Bidens biternata (Lour.) Merr. \& Sherff, 307 oligoflora (Klatt) Wild, 427

Bignoniaceae, 291 App., 387

Biogeography, 443-482*

Biophytum dendroides (Kunth) DC., 294 App.

Blanchetiodendron blanchetii (Benth.) Barneby \& J.W.Grimes, 388

Blechnaceae, 296 App.

Blechnum serrulatum Rich., 278, 282, 296 App.

Blepharis ciliaris (L.) B.L.Burtt, 193 App., 203, 214 App.

Blepharispermum hirtum Oliver, 306, 312

Blepharocalyx salicifolius (Kunth) O.Berg, 386

Bletia purpurea (Lam.) DC., 278, 294 App.

Blumea mollis (D.Don) Merr., 308

Bocagea longipedunculata Mart., 388

Boerhavia elegans Choisy, 196 App. repens L., 222 App. 
Bombacaceae, 331 Rev.

Bonnetiaceae, 329 Rev.

Book REVIEWs

Flora Malesiana, Series I. Volume 19. Cucurbitaceae (W.J.J.O. de Wilde \& B.E.E. Duyfjes), 327-328

Flora of Peninsular Malaysia, Series I: Ferns and Lycophytes, Volume 1. Malayan Forest Records No. 48 (B.S. Parris et al. eds), 328-330

Flora of Peninsular Malaysia, Series II: Seed Plants, Volume 1. Malayan Forest Records No. 49 (R. Kiew et al. eds), 328-330

Floral Diagrams: An Aid to Understanding Flower Morphology and Evolution (L.P. Ronse De Craene), 157-158

Natural and Cultural History of the Golfo Dulce Region, Costa Rica

(A. Weissenhofer et al. eds), 330-331

Seeds of Amazonian Plants (F. Cornejo \& J. Janovec), 325-327

Trees of Panama and Costa Rica (R. Condit, R. Pérez \& N. Daguerre), 483-484

Boraginaceae, 159-163*, 194 App., 215 App.

tribe Cynoglosseae, 159

Borneo, 450-451 Map, 453, 456

Boscio-Commiphoretalia, 306

Boscio-Commiphoretea abyssinicae, 305-306, 315

Bothriochloa insculpta (Hochst.) A.Camus, 305, 313-315

Bowdichia

virgilioides Kunth, 386, 391, 406-407, 409, 414

Brachiaria cruciformis (Sm.) Griseb., 308 lata (Schumach.) C.E.Hubb., 308 ramosa (L.) Stapf, 308

Brachypodium distachyum (L.) P.Beauv., 219 App.

Brassica tournefortii Gouan, 215 App.

Brassicaceae, 165-171*, 194 App., 215 App.

Brazil, 139-155, 373-400 [376 Map], 401-418 [404 Map]

Minas Gerais, 33-38*
Breteler, F.J., 343-350

Bridgewater, S.G.M., 273-296

Bromus

danthoniae Trin. ex C.A.Mey., 219 App.

fasciculatus C.Presl, 219 App.

madritensis L., 219 App.

rubens L., 219 App.

tectorum L., 219 App.

Bucanetes githagineus Lichtenstein, 190

Buchenavia hoehneana N.F.Mattos, 388

Buchnera hispida Buch.-Ham. ex D.Don, 307 paucidentata Engl. ex Skan, 431

Buglossoides arvensis (L.) I.M.Johnst., 215 App.

Bulbillaria gageoides Zucc., 48, 53

Burseraceae, 194 App., 387-388, 424, 427

Byrsonima coccolobifolia Kunth, 407 crassa Nied., 407 crassifolia (L.) Kunth, 274, 280-282, 285, 293 App.

sericea DC., 386

Cabralea canjerana (Vell.) Mart., 386, 391

Cactaceae, 157 Rev., 388

Cajamarca, Peru, 177-182*

Cakile arabica Velen. \& Bornm., 206, 215 App.

Caldcluvia D.Don, 41

Calea jamaicensis (L.) L., 291 App. trichotoma Donn.Sm., 278, 291 App.

Calendula arvensis L., 206, 217 App. tripterocarpa Rupr., 217 App.

Calliandra asplenioides (Nees) Renvoize, 386 houstoniana (Mill.) Standl., 280, 292 App.

Calligonum, 209 comosum L’Hér., 206, 223 App. crinitum Boiss. subsp. arabicum (Soskov) Soskov, Callipeltis 188-190 [188 Fig.], 192, 197 App.

cucullaris (L.) DC., 223 App. 


\section{Callisthene}

fasciculata Mart., 403, 412-413, 415

Calolisianthus (Griseb.) Gilg, 139-155* amplissimus (Mart.) Gilg, 139-147 [141

Fig., 144 Fig., 147 Fig.], 149-152

[149-150 Figs]

pendulus (Mart.) Gilg, 139-152 [141 Fig.,

144 Fig., 147-150 Figs]

speciosus (Cham. \& Schltdl.) Gilg,

139-152 [141 Fig., 144 Fig., 147-150

Figs]

Calophyllum

brasiliense Cambess., 287, 292 App., 386

Calycanthus, $158 \mathrm{Rev}$.

Calyptranthes

lindeniana O.Berg, 294 App.

Campanula L., 353, 355

Campanulaceae, 216 App., 291 App., 351-371

subfam. Campanuloideae, 366

subfam. Lobelioideae, 355, 366

tribe Rhigiophylleae Eddie \& Cupido, 352

Campo rupestre, Brazil, 33-38*, 139-155

Campomanesia laurifolia Gardner, 387

Camptonotus quadriceps, 444-445

Campylospermum umbricola (Tiegh.) Farron, 431

Canis

lupus arabs Pocock, 190, 208

Canscora

concanensis C.B.Clarke, 307

Capellenia Teijsm. \& Binn., 443, 461

moluccana Teijsm. \& Binn., 444, 461, 470, 472

Capparaceae, 194 App., 216 App.

Capparis

decidua (Forssk.) Edgew., 216 App.

sinaica Veill. ex Duhamel, 194 App.

spinosa L., 194 App., 216 App.

var. mucronifolia, 205

Capra

ibex nubiana F.Cuvier, 208

Capsella

bursa-pastoris (L.) Medik., 215 App.

Caracal

caracal schmitzi Matschie, 208

Carara Medik., 166
Cardamon (DC.) Fourr., 166

sativum (L.) Fourr., 166

Cardaria Desv., 165-166

draba (L.) Desv., 166, 215 App.

Cardiopetalum calophyllum Schltdl., 406-407

Carduus pycnocephalus L., 217 App.

Carissa spinarum L., 309

Carpinus, 158 Rev.

Carpotroche brasiliensis (Raddi) Endl., 388

Carrichtera аппиа (L.) DC., 215 App.

Carthamus nitidus Boiss., 217 App. oxyacantha M.Bieb., 217 App. oxycantha Boiss., 217 App.

Caryocar, 406 brasiliense Cambess., 407, 409

Caryophyllaceae, 194 App., 216 App.

Casearia arborea (Rich.) Urb., 386

eichleriana Sleumer, 386

sylvestris Sw., 295 App., 386

tremula (Griseb.) Griseb. ex C.Wright, 295 App.

Casuarinaceae, 329 Rev.

Catanduva, Brazil, 402

Caylusea hexagyna (Forssk.) M.L.Green, 223 App.

Cecropia pachystachya Trécul, 408

Cedrela odorata L., 287

Ceiba pentandra, $331 \mathrm{Rev}$.

Celastraceae, 292 App., 312, 387

Celtis, 469

Cenchrus ciliaris L., 195 App., 219 App. pennisetiformis Hochst. \& Steud., 219 App.

Centaurea

dhofarica Baker, 308, 312

eryngioides Lam., 205, 217 App.

mesopotamica Bornm., 217 App.

pseudosinaica Czerep., 194 App., 217 App. sinaica DC., 217 App. 


\section{Centaurium}

erythraea Rafn, 209, 219 App.

pulchellum (Swartz) Druce, 219 App.

Central Africa, 423-442*

Central America, 273-296

Centropodia forskaolii (Vahl) Cope, 195 App.

fragilis (P.Guinet \& Sauvage) Cope, 189,

Cereus 195 App., 219 App.

$$
\text { jamacaru DC., } 388
$$

Ceriscoides

curranii (Merr.) Tirveng., 29

Ceropegia

bulbosa Roxb., 309

Cerradões, Brazil, 139-155, 401-418

Ceylon, 339

Chamaecrista

diphylla (L.) Greene, 278, 292 App.

Chamaedorea, 273, 284, 287

Chase, M.W., 43-59

Chassalia, 424

Chazaliella, 424

Checklists

flora of Gabon, 423-442*

plant taxa from Jabal Aja', 214-224 App.

plant taxa from 'Uruq Bani Ma'arid, 193-197 App.

Cheilanthes

acrostica (Balb.) Tod., 205, 223 App.

pteridioides (Reichard) C.Chr., 223 App. vellea (Aiton) F.Muell., 205, 223 App.

Chenopodium murale L., 215 App.

China, 269-272*, 297-300

South East, 450-451 Map

Xizang, 61-109*

Chiquibul Forest Reserve, Belize, 273-296 [275 Map]

Chlamydotis macqueenii J.E.Gray, 208

Chloranthaceae, 329 Rev., 391

Chrozophora oblongifolia (Delile) A.Juss. ex Spreng., 195 App.

tinctoria (L.) A.Juss., 195 App., 219 App.

Chrysanthemum coronarium L., 217 App.

Chrysobalanaceae, 387

Chrysophyllum imperiale (Linden ex Koch \& Fintelm.)

Benth. \& Hook.f., 388

Chrysopogon

macleishii Cope, 309, 312

plumulosus Hochst., 195 App.

Cibotiaceae, 329 Rev.

Cistaceae, 217 App.

Cistanche

phelypaea (L.) Cout., 196 App., 222 App.

Citation, of Desmos, 173-175

Citharexylum

caudatum L., 280-281, 295 App.

Citrullus

colocynthis (L.) Schrad., 195 App., 202, 218 App.

Cleistachne

sorghoides Benth., 309

Cleomaceae, 194 App., 217 App.

Cleome

amblyocarpa Barratte \& Murb., 187, 194

App., 217 App.

rupicola Vicary, 205, 217 App.

Clethra

occidentalis (L.) Kuntze, 280-281, 292

App.

scabra Pers., 386, 391

Clethraceae, 292 App., 329 Rev., 391

Clidemia capitellata (Bonpl.) D.Don, 293 App.

sericea D.Don, 293 App.

Clusia

flava Jacq., 282, 292 App. quadrangula Bartlett, 292 App.

Clusiaceae, 292 App., 387

Clypeola aspera (Grauer) Turrill, 209, 215

App.

jonthlaspi L., 216 App.

Coccocypselum hirsutum Bartl. ex DC., 295 App.

Cocculus pendulus (J.R.Forst. \& G.Forst.) Diels, 196 App.

Cochlearia coronopus L., 166

nilotica Delile, 166

Coelachne, 425 africana Pilg., 431

Cola umbratilis Brenan \& Keay, 348 
Colchicaceae, 217 App.

Colchicum ritchii $\mathrm{R}$.Br., 206, 217 App.

Combretaceae, 292 App., 312, 348, 388

Combretum tarquense J.J.Clark, 348

Cometia lucida Baill., 343

Commelina albescens Hassk., 308

Commiphora, 315 gileadensis (L.) C.Chr., 306 kua (J.F.Royle) Vollesen, 306 leptophloeos (Mart.) J.B.Gillet, 388 myrrha (Nees) Engl., 187, 194 App.

Compositae, 194 App., 217 App. see also Asteraceae

Connarus suberosus Planch., 406, 408

Conostegia icosandra (Sw. ex Wikstr.) Urb., 293 App. xalapensis (Bonpl.) D.Don ex DC., 293 App.

Conservation of Gardenia, 11-32* in Saudi Arabia, 183-197*, 199-224*

Convolvulaceae, 195 App., 218 App., 424, 427

Convolvulus

arvensis L., 218 App., 308 asyrensis Kotschy, 195 App. auricomus (A.Rich.) Bhandari var. auricomus, 195 App. buschiricus Bornm., 195 App. oxyphyllus Boiss. subsp. oxycladus Rech.f., 205, 218 App.

pilosellifolius Desr., 205, 218 App.

prostratus Forssk., 308

Conyza

bonariensis (L.) Cronquist, 217 App.

Copaifera langsdorffii Desf., 386, 391, 393, 409

Corbichonia decumbens (Forssk.) Exell, 188, 196 App.

Corchorus trilocularis L., 309

Cordia crenata Delile subsp. crenata, 309
Cordiera

concolor (Cham.) Kuntze, 386

elliptica (Cham.) Kuntze, 386

Cornulaca arabica Botsch., 188-190, 193 App.

Coronopus Mill., 166

Coronopus Zinn, 165-166

ruellii All., 166

Corrections, to flora of Gabon, 423-442

Corvus ruficollis Lesson, 190

Cosentina vellea (Aiton) Tod., 223 App.

Costaceae, 292 App.

Costus pulverulentus C.Presl, 283, 292 App.

Côte d'Ivoire, 343-350*

Cotyliscus Desv., 166 niloticus (Delile) Desv., 166

Crassula alata (Viv.) A.Berger, 218 App.

Crassulaceae, 218 App.

Craterocapsa Hilliard \& B.L.Burtt, 359, 365-367, 369

montana (A.DC.) Hilliard \& B.L.Burtt, 354, 357, 360 Fig., 368

tarsodes Hilliard \& B.L.Burtt, 354, 357, 365,368

Crematogaster

borneensis, 445

Crepidospermum

atlanticum Daly, 387

Crepis, 205

aspera L., 217 App.

kotschyana (Boiss.) Boiss., 217 App.

sancta (L.) Bornm., 217 App.

Crescentia

cujete L., 278, 280, 285, 291 App.

Critoniopsis leiocarpa (DC.) H.Rob., 278, 282, 291 App.

Crossopetalum, 292 App.

Crotalaria aegyptiaca Benth., 196 App. leptocarpa Balf.f., 196 App. ononoides Benth., 429 retusa $\mathrm{L} .$, 307, 313

Croton L., 33-34 sect. Medea (Klotzsch) Baill., 33-38* arlineae D.Medeiros et al., 34 
bilbergianus Müll.Arg. subsp. pyramidalis (Donn.Sm.)

G.L.Webster, 278

gnidiaceus Baill., 34

josephinus Müll.Arg., 33-38* [35 Fig., 36]

pradensis D.Medeiros et al., 34

pyramidalis Donn.Sm., 278

vestitus Spreng., 34

Cruciferae, 329 Rev.

Crypsinus

cruciformis (Ching) Tagawa, 266

oxylobus (Wall. ex Kunze) Sledge, 265

Cryptogrammaceae, 329 Rev.

Ctenolepis

cerasiformis (Stocks) Hook.f., 202, 218

App.

Ctenolophonaceae, 329 Rev.

Cucumis

prophetarum L., 218 App.

sativus L., 307

Cucurbitaceae, 195 App., 218 App., 327-328 Rev., 424, 428

Cunoniaceae, 157 Rev.

Cupania

paniculata Cambess., 386

rufescens Triana \& Planch., 278

Cuphea

appendiculata Benth.

var. appendiculata, 293 App.

aristata Hemsl., 278, 293 App.

CupIDo, C.N., 351-371

Curatella

americana L., 274, 407

Cuscuta planiflora Ten., 218 App.

Cutandia memphitica (Spreng.) K.Richt., 219 App.

Cyathea, 296 App.

corcovadensis (Raddi) Domin, 386

delgadii Sternb., 386

phalerata Mart., 386

villosa Willd., 386

Cyatheaceae, 296 App.

Cyathogyne Müll.Arg., 343-350*

bussei Pax, 343-344

grandifolia Pax \& K.Hoffm., 345

spathulifolia Pax, 343

viridis Müll.Arg., 343-344, 349

Cybianthus

glaber A.DC., 386
Cybistax

antisyphilitica (Mart.) Mart., 408

Cyclocodon Griff. ex Hook.f., 353

Cymbopetalum

brasiliense (Vell.) Benth. ex Baill., 174

Cymbopogon

commutatus (Steud.) Stapf, 203, 205, 219

App.

schoenanthus (L.) Spreng., 202-203, 205, 219 App.

Cynodon

dactylon (L.) Pers., 219 App.

Cynomoriaceae, 195 App., 218 App.

Cynomorium

coccineum L., 195 App., 218 App.

Cyperaceae, 195 App., 218 App., 292 App., 424,428

Cyperus

alulatus J.Kern, 309

aucheri Jaub. \& Spach, 195 App.

conglomeratus Rottb., 206, 218 App.

var. aucheri (Jaub. \& Spach)

C.B.Clarke, 195 App.

longus L., 309

macrorrhizus Nees, 189, 195 App.

squarrosus L., 308

Cyphia

comptonii, 368

Cyphostemma

ternatum (Forssk.) Desc., 306

Cyrtocarpa

caatingae J.D.Mitch. \& Daly, 388

Dacrydium Lamb., 41

Dacryodes osika (Guillaumin) H.J.Lam, 427

yangambiensis Louis ex Troupin, 427

Dalbergia, 460

miscolobium Benth., 386, 389

violacea (Jacq.) Hoffmanns., 408

Daphniphyllaceae, 329 Rev.

Datiscaceae, 329 Rev.

Dauby, G., 423-442

Davilla

elliptica A.St.-Hil., 407

Delgado, M.N., 139-155

Dendroctonus, 273, 284, 287

Dennstaedtiaceae, 296 App.

Desmodio gangetico-Themedetum quadrivalvis, 301, 305, 312, 314, 316 


\section{Desmodium}

gangeticum (L.) DC., 305, 312

Desmos Lour., 173-175*

viridiflorus (Bedd.) Saff., 173

viridiflorus Saff., 173-175 [174]

Dessein, S., 423-442

Diascia, 158 Rev.

Dichanthium

annulatum (Forssk.) Stapf, 308

foveolatum (Delile) Roberty, 195 App.

Dichapetalaceae, 348

Dicranopteris pectinata (Willd.) Underw., 282, 296 App.

Dictyoloma vandellianum A.Juss., 386

Digitaria ciliaris (Retz.) Koeler, 309

stricta Roth ex Roem. \& Schult., 308

Dilodendron

bipinnatum Radlk., 408, 413

Dimeria ornithopoda Trin., 309

Dimorphandra mollis Benth., 408

Dioscoreophyllum gossweileri Exell, 431

Diospyros L., 339-341* sect. Patonia Hiern, 340

burchellii Hiern, 408

hispida A.DC., 407

ketun B.Walln., 392

undulata Wall. ex G.Don var. walkeri (Wight) Bakh., 340

walkeri (Wight) Gürke, 339-340

Dipcadi erythraeum Webb \& Berth., 196 App. unicolor (Stocks) Baker, 196 App.

Diplotaxis acris (Forssk.) Boiss., 202, 205, 216 App. erucoides (L.) DC., 216 App.

Diplycosia Blume, 39-42* apoensis Elmer, 42 bartolomei Ferreras \& Argent, 39-42

[40 Fig.]

gracilipes J.J.Sm., 42

heterophylla Blume, 42

loheri Merr., 42

luzonica (A.Gray) Merr., 39, 42

parvifolia Merr., 42

paulsmithii Argent, 42 trinervia Elmer, 42

Dipsacaceae, 219 App.

Dipterygium glaucum Decne., 187-189, 194 App.

Dipteryx alata Vogel, 406-407, 409

Dischistocalyx, 424 champluvierianus Lejoly \& Lisowski, 425

Discospermum whitfordii (Elmer) S.J.Ali \& Robbrecht, 30

Ditta Griseb., 444

Dodonaea viscosa (L.) Jacq., 278, 295 App., 307

Dolichos, 424-425 subcapitatus R.Wilczek, 429

Dorstenia foetida (Forssk.) Schweinf., 309, 311

Drimys brasiliensis Miers, 386, 391

Dryopteridaceae, 428

Ducrosia anethifolia (DC.) Boiss., 224 App.

Duguetia chrysocarpa Maas, 388

Dyschoriste dalyi A.G.Mill., 309, 312

Dysoxylum, 29

Dystrophic cerradão, 401-418*

Ebenaceae, 339, 392

Echinochloa, 195 App.

Echinops, 217 App. mandavillei Kit Tan, 203, 207, 209, 217 App.

Echium, 194 App. arabicum R.Mill, 215 App. rauwolfii Delile, 215 App.

Eclipta prostrata (L.) L., 309

Ecology, in relation to seed types, 351-371

Ecpoma geanthum (Hiern) N.Hallé, 435

EdDIE, W.M.M., 351-371

Eichhornia, 158 Rev.

Elaeocarpaceae, 157 Rev.

Elaeocarpus L., 41

Elaphoglossum cinnamomeum (Baker) Diels, 424, 428

kuhnii Hieron., 424, 428

plumosum (Fée) T.Moore, 428 


\section{Eleocharis}

uniglumis (Link) Schult., 205-206, 209, 218 App.

Elionurus, 424 hirtifolius Hack., 432

Emendation, in Pedicularis, 61-109*

Emex spinosa (L.) Campd., 223 App.

Emmotum nitens (Benth.) Miers, 386, 391, 401, 403, 406-407

Empty Quarter, Saudi Arabia, 183-197* [184 Map]

Encyclia michuacana (La Llave \& Lex.) Schltr., 278, 294 App.

Endemism in Oman, 301-319*

in Saudi Arabia, 183-197*

Endespermum Blume

Endospermum Benth., 443-482* [460], \& Figs, \& Maps subgen. Capellenia (Teijsm. \& Binn.) Pax \& K.Hoffm., 444, 461

subgen. Endospermum, 444

subgen. Euendospermum Pax \&

K.Hoffm., 444, 461

banghamii Merr., 443, 447-448 [447 Fig.], 476-477

beccarianum Pax \& K.Hoffm., 465-466 var. crassirameum Pax \& K.Hoffm., 465-466

var. tenuirameum $\operatorname{Pax} \&$ K.Hoffm., 465 borneense auct., 465

borneense Benth. ex Müll.Arg., 464

chinense Benth., 443, 449-450, 452-453, 456, 459, 461

var. malayanum Pax \& K.Hoffm., 465

diadenum (Miq.) Airy Shaw, 445, 447-448, 450, 452-457 [454 Fig., 455

Map], 459, 463-464, 467, 478, 480

domatiophorum J.Schaeff., 445-448 [446 Fig.], 450, 452-453, 456 \& Map, 463, 467-468, 470, 480

eglandulosum Pax \& K.Hoffm., 478

formicarum Becc., 470

labios Schodde, 470, 472

subsp. gracilipes Schodde, 470

subsp. labios, 470 macrophyllum Pax \& K.Hoffm., 449-453, 456, 459

malaccense Benth. ex Müll.Arg., 464

malayanum (Pax \& K.Hoffm.) Chatterjee, 465

medullosum L.S.Sm., 445, 447-448 [447

Fig.], 450-453, 456-457 [457 Map], 459, 464, 468, 470, 474, 480

moluccanum (Teijsm. \& Binn.) Kurz, 444 445, 447-448, 450, 452-453, 457-459 [458 Fig., 459 Map], 461, 463, 470, 472-474, 480

myrmecophilum L.S.Sm., 444-446 [446

Fig.], 448, 450-453, 460 Map, 463, 470, 472-473, 480

ovalifolium Pax \& K.Hoffm., 465

ovatum Merr., 445, 447-448, 450, 452-453, 456, 459, 461 Map, 464, 474, 480

peltatum Merr., 445, 447-448 [447 Fig.], 450, 452-453, 456-457, 459, 462 Мap, 464, 467, 475, 480

philippinense Elmer ex Merr., 475

quadriloculare Pax \& K.Hoffm., 443, 445-448 [446 Fig.], 450, 452-453, 456-457, 461 Map, 464, 467, 476-478, 480

ronaldii J.Schaeff., 443, 448, 476-477

EndRess, P.K., 157-158 Rev.

Engelhardtia Lesch. ex Blume, 41

Enicostemma axillare (Lam.) A.Raynal, 309

Enneapogon desvauxii P.Beauv., 195 App., 219 App.

persicus Boiss., 219 App.

schimperianus (Hochst. ex A.Rich.)

Renvoize, 219 App.

Enterolobium

gummiferum (Mart.) J.F.Macbr., 389, 408

Ephedra

foliata Boiss. ex C.A.Mey., 187, 195

App., 202, 205-206, 219 App.

Ephedraceae, 195 App., 219 App.

Epitype, of Pedicularis, 111-138*

Equisetaceae, 329 Rev.

Eragrostis

barrelieri Daveau, 220 App.

maypurensis (Kunth) Steud., 294 App.

viscosa (Retz.) Trin., 309

Eremanthus 
glomerulatus Less., 408

Eremobium

aegyptiacum (Spreng.) Asch. \& Schweinf. ex Boiss., 194 App., 206, 216 App.

Ericaceae, 39-42*, 419-422*

Eriocaulaceae, 257-263*, 424, 428

Eriocaulon L., 257-263

ansarii Pradeep \& Sunil, 257, 260, 262-263

baramaticum Shimpale et al., 257

epedunculatum Potdar et al., 257

malabaricum Pradeep \& Nampy,

257-263* [258-259 Figs]

pykarense Nampy \& Manudev, 257-263*

[259 Fig., 260, 261 Fig.]

sollyanum Royle, 257, 260, 262-263

trilobum Buch.-Ham. ex Körn., 263

Eriosema, 424

pellegrinii Tisser., 429

Eriotheca gracilipes (K.Schum.) A.Robyns, 407 macrophylla (K.Schum.) A.Robyns, 387 pubescens (Mart. \& Zucc.) Schott \& Endl., 408

Erodium

laciniatum (Cav.) Willd., 219 App. neuradifolium Delile ex Godr., 219 App. touchyanum Delile ex Godr., 219 App.

Eruca sativa Mill., 216 App.

Erysimum

hedgeanum Al-Shehbaz, 201, 207, 209, 216 App.

Erythroxylaceae, 329 Rev., 389, 391

Erythroxylum

daphnites Mart., 389, 407

suberosum A.St.-Hil., 408

vaccinifolium Mart., 386, 391

Esenbeckia

grandiflora Mart., 386

Espinhaçao Range, Brazil, 373-400*

Euclea [376 Map]

schimperi (A.DC.) Dandy, 307

Eugenia, 29

capuli (Schltdl. \& Cham.) Hook. \& Arn., 294 App.

florida DC., 386

klotzschiana O.Berg, 389

laruotteana Cambess., 392 punicifolia (Kunth) DC., 386

winzerlingii Standl., 278, 294 App.

Euphorbia

chamaepeplus Boiss. \& Gaill., 219 App.

granulata Forssk., 219 App.

hirta L., 219 App.

indica Lam., 307

peplus L., 219 App.

schimperiana Scheele, 219 App.

Euphorbiaceae, 33-38, 195 App., 219 App.,

292 App., 325 Rev., 343-350*, 388,

391, 443-482*

subfam. Crotonoideae, 444

tribe Adenoclineae, 444

tribe Crotoneae, 443

tribe Gelonieae, 444

subtribe Endosperminae, 444

Euplassa

semicostata Plana, 392

Eustachys

paspaloides (Vahl) Lanza \& Mattei, 305, 313-314

Exacum

arabicum Thulin, 306, 312

Exallage, 424-425

auricularia (L.) Bremek., 433

Extrafloral nectaries, in Calolisianthus, $139-155^{*}$

Fabaceae, 157 Rev., 429, 460, 469

subfam. Caesalpinioideae, 292 App.

subfam. Mimosoideae, 292 App.

Fagaceae, 292 App.

Fagonia

bruguieri DC., 206, 224 App.

glutinosa Delile, 224 App.

indica Burm.f., 187, 189, 197 App., 224

App.

var. schweinfurthii Hadidi, 197 App.

schweinfurthii (Hadidi) M.Hall, 183, 197

App.

Falco

cherrug Gray, 208

pelegrinoides Temminck, 191

Faramea

nigrescens Mart., 386

Farsetia, 188

aegyptia Turra, 205-206, 216 App.

burtoniae Oliv., 188-189, 194 App.

dhofarica, 194 App. 
longisiliqua Decne., 188-189, 194 App. stylosa R.Br., 194 App.

Felis

margarita harrisoni Hemmer, Grub \&

Groves, 189

FERreras, U.F., 39-42

Ferula

communis L., 224 App.

Ficus

subgen. Ficus, 269

sect. Ficus, 269

subsect. Frutescentiae Sata, 269

bistipulata Griff., 270-271

congesta H.Lév. \& Vaniot, 271

cuneata H.Lév. \& Vaniot, 269-271

var. congesta H.Lév. \& Vaniot, 271

enormis (Mart. ex Miq.) Mart., 408

erecta Thunb., 269-270, 272

gasparriniana Miq., 269-270

var. viridescens (H.Lév. \& Vaniot)

Corner, 271

heteromorpha Hemsl., 269-271

neriifolia Sm., 270

palmata Forssk., 221 App.

subsp. virgata (Roxb.) Browicz, 205, 221 App.

pandurata Hance, 269-270

pedunculosa, 269-272*

pertusa L.f., 386

stapfii H.Lév., 269-271

sycomorus L., 309

trivia Corner, 269-272 [270]

var. laevigata S.S.Chang, 270, 272

var. tenuipetiolata S.S.Chang, 269

tuphapensis Drake, 269-270

vasta Forssk., 309

Fiji, 451 Map, 453, 456, 459

Filago

desertorum Pomel, 217 App.

Fimbristylis

bisumbellata (Forssk.) Bubani, 308

splendida C.B.Clarke, 428

Flacourtiaceae, 325 Rev.

Flaveria trinervia (Spreng.) C.Mohr, 217 App.

Flora

of Gabon, 423-442*

of Indonesia, 419-422*

of the Philippines, 39-42*

Floresta xeromorfa, Brazil, 402
Floristic survey, 401-418*

Floristics, of the San Pastor Savanna, Belize, 273-296*

Flueggea virosa (Roxb. ex Willd.) Royle, 306

Fog-oasis, 301-319*

Forests, Atlantic Semideciduous, $373-400^{*}$

Forsskaolea tenacissima L., 224 App.

Forsteronia myriantha Donn.Sm., 291 App.

Fumaria parviflora Lam., 222 App.

Furley, P.A., 273-296

Fusarium, 330 Rev.

Fynbos, South Africa, 363-365

Gabon, flora of, 423-442*

Gagea Salisb., 43-59* sect. Didymobulbos K.Koch, 52 sect. Plecostigma Pascher, 52 afghanica A.Terracc., 43, 45, 49, 51-57

[54, 56 Fig.]

amblyopetala Boiss. \& Heldr. var. bulbifera Boiss., 51 circumplexa Vved., 221 App. dayana Chodat \& Beauverd var. conjugens (Pascher) Heyn \& Dafni, 221 App.

dschungarica Regel, 43, 45, 49-53

[50 Fig.], 55-57 [56 Fig.]

ebulbillosa (Boiss.) Levichev, 54

gageoides (Zucc.) Vved., 43, 45, 47-53

[50 Fig.], 55-57

graeca (L.) A.Terracc., 47

kashmirensis Turrill, 43, 52-54

kunawurensis (Royle) Greuter, 43-59

[50 Fig., 54]

ova Stapf, 47, 54

perpusilla Pascher, 44

persica Boiss., 43, 47-48, 50-53

var. ebulbillosa Boiss., 51, 54

var. kashmirensis (Turrill) S.Dasgupta

\& Deb, 51-52, 54

var. persica, 51

reticulata (Pall.) Schult. \& Schult.f., 44, 202, 221 App.

serotina (L.) Ker-Gawl., 43

setifolia Baker, 44 
stipitata Merckl. ex Bunge, 43, 46-47, $51-52,54$

tehranica Gand., 44

tenera Pascher, 43, 46, 49, 52-57 [54, 56

Fig.]

trinervia (Viv.) Greuter, 47

Galium

ceratopodum Boiss., 223 App.

setaceum Lam., 205, 223 App.

Gardenia J.Ellis, 11-32*

acutifolia Elmer, 29

barnesii Merr., 11, 13-14 [14 Fig.], 16-17

[16 Map], 29-30

carinata sensu Fern.-Vill., non Wall. ex

Roxb., 30

curranii Merr., 29

elata Ridl., 11, 13, 18, 20

var. elata, 13, 19-21 [19 Fig.]

var. longipedicellata K.M.Wong, 13, 19, 21 [21 Fig.]

elliptica Elmer, 29

glutinosa auct., non Teijsm. \& Binn., 18, 20-21

glutinosa Teijsm. \& Binn., 20

jasminoides J.Ellis, 11, 17

lagunensis Merr., 29

lobbii Craib, 18

longiflora S.Vidal, 18

longituba Ridl., 18

megalocarpa Merr., 14

merrillii Elmer, 29

morindifolia Elmer, 30

mutabilis Reinw. ex Blume, 11-13, 22-25

[23 Fig., 24 Map]

negrosensis Merr., 30

obscura (Blanco) S.Vidal, 30

obscurinervia Merr., 30

ornata K.M.Wong, 11, 13, 24-26 [24

Map, 25, 26 Fig.]

pinnata (Blanco) Merr., 30

pseudopsidium (Blanco) Fern.-Vill., 11, 28

pubifolia Merr., 30

ramosa Merr., 22

segmenta Elmer, 14

speciosa Hook.f., 18

subcarinata (Corner) Y.W.Low, 25-26

thunbergia sensu Fern.-Vill., 30

tubifera auct., non Wall. ex Roxb., 18

tubifera Wall. ex Roxb., 21, 26, 28

var. tubifera f. elata (Ridl.) K.M.Wong, 18

vulcanica K.M.Wong, 11, 13, 24 Map, 26,

27 Fig.

whitfordii Elmer, 30

Gastrocotyle

hispida (Forssk.) Bunge, 194 App., 215 App.

Gaylussacia

brasiliensis (Spreng.) Meisn., 386

Gazella

gazella Pallas, 189

subgutturosa marica Güldenstädt, 189

Generic delimitation, of Thecacoris, 343-350

Genipa infundibuliformis Zappi \& Semir, 388

Gentianaceae, 139-155, 219 App., 312, 430

tribe Chironieae, 139

tribe Exaceae, 139

tribe Gentianeae, 139

tribe Helieae, 139-155

tribe Potalieae, 139

tribe Saccifolieae, 139

Gentianales, 139

Geographic distribution, 373-400*

Geonoma brevispatha Barb.Rodr., 386

Geophila

ingens Wernham, 433

lancistipula Hiern, 434

obvallata (Schumach. \& Thonn.) F.Didr., 433

repens (L.) I.M.Johnst., 433-434

Geraniaceae, 195 App., 219 App.

Geranium

biuncinatum Kokwaro, 219 App.

mascatense Boiss., 219 App.

molle L., 219 App.

rotundifolium L., 219 App.

Gesneriaceae, 331 Rev.

Ghana, 343-350*

GibBy, M., 328-330 Rev.

Gironniera subaequalis Planch., 323

Gisekia

pharnaceoides L., 195 App.

Gisekiaceae, 195 App.

Gladiolus

candidus (Rendle) Goldblatt, 309

italicus Mill., 205, 209, 220 App.

Gleicheniaceae, 248, 296 App. 


\section{Gliricidia}

sepium (Jacq.) Kunkun ex Walp., 292 App.

Glossonema varians (Stocks) Hook.f., 194 App.

Gloxinia perennis, $331 \mathrm{Rev}$.

Gochnatia hatschbachii Cabrera, 392

polymorpha (Less.) Cabrera, 386

Goiás State, Brazil, 401-418 [404 Map]

Gomphocarpus, 424

fruticosus subsp. setosa (Forssk.) Goyder \& Nicholas, 309

sinaicus Boiss., 215 App.

swynnertonii (S.Moore) Goyder \&

Nicholas, 426

Gomphogyne, 327 Rev.

Goodwin, Z.A., 273-296

Gramineae, 195 App., 219 App., 309

see also Poaceae

Grammitidaceae, 329 Rev.

Grammitis

ebenina (Maxon) Tardieu, 432

nigrocincta Alston, 432

Great Nicobar Island, 321-324*

Guapira, 408

graciliflora (Schmidt) Lundell, 386, 408

noxia (Netto) Lundell, 408

opposita (Vell.) Reitz, 386, 391, 393

Guatteria

notabilis Mello-Silva \& Pirani, 392

rupestris Mello-Silva \& Pirani, 386, 392

sellowiana Schltdl., 386

Guettarda

combsii Urb., 295 App.

tikalana Lundell, 295 App.

Gunillaea Thulin, 353

Gymnarrhena micrantha Desf., 217 App.

Gymnocarpos decandrus Forssk., 202, 205-206, 216 App.

Gynandiris, 205

sisyrinchium (L.) Parl., 220 App.

Gynerium

sagittatum (Aubl.) P.Beauv., 282-283, 294 App.

Gynotroches Blume, 41

Gyps

fulvus Hablizl, 208
Gypsophila

capillaris (Forssk.) C.Chr., 202, 216 App.

viscosa Murray, 216 App.

Hackelochloa

granularis (L.) Kuntze, 308

HaLL, M., 183-197, 199-224

Haloragis, 158 Rev.

Halothamnus bottae Jaub. \& Spach, 193 App.

iraqensis Botsch., 215 App.

Haloxylon, 209

persicum Bunge ex Boiss. \& Buhse, 189, 193 App.

salicornicum (Moq.) Bunge ex Boiss., 187-189, 193 App., 202, 206, 215 App.

Hammada scoparia sensu Collenette, 193 App.

Hancornia speciosa B.A.Gomes, 408

Handroanthus ochraceus (Cham.) Mattos, 386 serratifolius (Vahl) S.O.Grose, 408

HAO, G., 297-300

Haplophyllum tuberculatum (Forssk.) A.Juss., 187, 197 App.

Harpullia Roxb., 1-9* subgen. Harpullia, 7 subgen. Otonychium, 7 mabberleyana W.N.Takeuchi, 1-9

[3 Fig. \& Map] ramiflora Radlk., 1 rhachiptera Radlk., 1-9 [3 Map, 5 Fig., 7]

HARRIS, D.J., 273-296

Haston, E., 483-484 Rev.

Haumaniastrum, 425 caeruleum (Oliv.) P.A.Duvign. \& Plancke, 431

Hedyosmum brasiliense Miq., 386, 391

Hedyotis, 433

Hedypnois rhagadioloides (L.) F.W.Schmidt, 217 App.

Heldreichia silaifolia Hook.f. \& Thomson, 170

Helianthemum aegyptiacum (L.) Mill., 217 App. kahiricum Delile, 205-206, 217 App. ledifolium (L.) Mill., 217 App. 
lippii (L.) Dum.Cours., 205, 217 App.

Helichrysum mechowianum Klatt var. ceres (S.Moore) Beentje, 427

Heliconia, 283, 292 App.

Heliconiaceae, 292 App.

Helicostylis tomentosa (Poepp. \& Endl.) Rusby, 387

Helicteres guazumifolia Kunth, 293 App.

Heliotropium crispum Desf., 194 App., 205, 215 App. digynum (Forssk.) C.Chr., 194 App. longiflorum (Hochst. ex A.DC.) Jaub. \& Spach, 309

ramosissimum (Lehm.) DC., 194 App., 205, 215 App.

rariflorum Stocks, 194 App.

Herbarium, in Madras, 173-175*

Hermannia paniculata Franch., 308

Hernandia sonora auct. non L., 470

Hernandiaceae, 157 Rev.

Herniaria hirsuta L., 216 App.

Heterocaryum szovitsianum (Fisch. \& C.A.Mey.) A.DC., 215 App.

Heterocentron subtriplinervium (Link \& Otto) A.Braun \& C.D.Bouché, 293 App.

Heteropogon contortus (L.) P.Beauv. ex Roem. \& Schult., 305, 313-315

Heteropterys byrsonimifolia A.Juss., 408

Hibiscus costatus A.Rich., 293 App. micranthus L., 205-206, 221 App.

Hicks, J., 273-296

Himalaya, 61-109* Sino-Himalaya, 111-138*

Himatanthus obovatus (Müll.Arg.) Woodson, 408

Hippocrepis constricta Kunze, 221 App. unisiliquosa $\mathrm{L}$. subsp. bisiliqua (Forssk.) Bornm., 221 App.
Hiraea

reclinata Jacq., 293 App.

Hirschfeldia incana (L.) Lagr.-Foss., 205, 216 App.

Hirtella glandulosa Spreng., 386, 403, 407

Holocalyx balansae Micheli, 389

Hordeum murinum $\mathrm{L}$. subsp. glaucum (Steud.) Tzvelev, 205, 220 App.

spontaneum K.Koch, 205, 220 App.

Hornungia persica (Boiss.) Rouy, 53

HovenkAmp, P., 265-267

Hu, C.M., 297-300

Hughes, M., 225-255

Humiria balsaminifera Aubl., 386

Hyacinthaceae, 196 App., 220 App.

Hyaena hyaena sultana Pocock, 208

Hybantho durae-Anogeissetum dhofaricae, 305,315

Hymenaea stigonocarpa Mart. ex Hayne, 386, 407

Hymenocardia acida, 437

Hymenophysa C.A.Mey. ex Ledeb., 166 pubescens C.A.Mey., 166

Hymenostegia gracilipes Hutch. \& Dalziel, 348

Hyoscyamus muticus L., 223 App. pusillus L., 223 App.

Hyparrhenia hirta (L.) Stapf, 205, 220 App.

Hypecoum pendulum L., 222 App.

Hypericaceae, 293 App.

Hyperico-Rhamnetea, 307

Hypericum terrae-firmae Sprague \& L.Riley, 293 App.

Hystrix indica Kerr, 208

Icacinaceae, 391

Ifloga spicata (Forssk.) Sch.Bip., 218 App. 
Ilex

dumosa Reissek, 386

guianensis (Aubl.) Kuntze, 281-282, 291 App.

lundii Warm., 386

Illiciaceae, 157 Rev., 329 Rev.

Illicium, 158 Rev.

Impatiens

balsamina L., 306

Important Plant Area (IPA), 183-197*, 199-224*

India, 173-175*, 257-263*, 339

Great Nicobar Biosphere Reserve, 321-324

North, 450-451 Map

Northwest, 111-138*

Indigofera, 424

congolensis De Wild. \& T.Durand

var. congolensis, 429

spinosa Forssk., 196 App.

tinctoria L., 309

Indonesia, 225-255

flora of, 419-422*

South and West Sulawesi, 225-255 [227 Map]

Inga, 326 Rev., 408

cocleensis Pittier, 281, 292 App.

punctata Willd., 292 App.

INGROUILLE, M.J., 43-59

Iphiona

scabra DC., 194 App.

Ipomoea

linosepala Hallier $\mathrm{f}$.

subsp. alpina (Rendle) Lejoly \& Lisowski, 427

Ips, 284

Iridaceae, 36, 220 App., 293 App.

Irlbachia

pendula (Mart.) Maas, 152

Isatis L., 166

lusitanica L., 205, 216 App.

Isolectotype, in Croton, 33, 36

Isparta, Turkey, 159-163*

Jabal Aja', Saudi Arabia, 199-224* [200

Map, 201 Fig., 203-204 Figs]

checklist of plant taxa from, 214-224 App.

conservation threats in, 211

lower plains of, 202

Nafud sands of, 206-207 Fig.

qualification as an IPA, 209-210 and SWC protected area system plan, 210

socio-economics of, 210

upper plains and slopes of, 203

Jacaratia, 326 Rev.

Jasminum

grandiflorum

subsp. floribundum (Fresen.)

P.S.Green, 306

JAYANTHI, J., 321-324

Joannesia

princeps Vell., 388

Juncaceae, 220 App.

Juncus

bufonius L., 220 App.

Juniperetea procerae, 306

Justicia

rottleriana Wallich, 336

Kandis Adans., 166

perfoliata (L.) Kerguélen, 166

Karoo, South Africa, 365

KaRthigeYAN, K., 321-324

Kashmir, 111-138*

Kasuya, M.C.M., 139-155

Kibara

coriacea (Blume) Tulasne, 323

Kickxia acerbiana (Boiss.) Täckh. \& Boulos, 222 App.

aegyptiaca (L.) Nábělek, 222 App.

pseudoscoparia V.W.Sm. \& D.A.Sutton, 209, 222 App.

Kielmeyera

coriacea Mart. \& Zucc., 407

petiolaris Mart., 386

Klaineanthus Pierre ex Prain, 444, 450, 452 gaboniae Pierre ex Prain, 449-450

Koelpinia linearis Pall., 206, 218 App.

Kohautia caespitosa Schnizl., 197 App.

Kotschya, 425 ochreata (Taub.) Dewit \& P.A.Duvign. var. ochreata, 430

Labiatae, 196 App., 220 App.

LACHENAUD, O., 423-442

Lacistema aggregatum (P.J.Bergius) Rusby, 280, 293 App. 
Lacistemataceae, 293 App.

Laetia thamnia L., 295 App.

Lafoensia pacari A.St.-Hil., 408 vandelliana Cham. \& Schltdl., 386

Lallemantia royleana (Benth.) Benth., 209, 220 App.

Lamanonia ternata Vell., 386

Lamarckia aurea (L.) Moench, 220 App.

Lamiaceae, 196 App., 220 App., 293 App., 424,431

Lappula sinaica (A.DC.) Asch. \& Schweinf., 215 App. spinocarpos (Forssk.) Asch. ex Kuntze, 215 App.

Lasiopogon muscoides (Desf.) DC., 218 App.

Lasiospermum brachyglossum DC., 218 App.

Lasiurus scindicus Henrard, 188-189, 195 App.

Launaea crassifolia (Balf.f.) C.Jeffrey, 306 fragilis (Asso) Pau, 218 App. mucronata (Forssk.) Muschl., 194 App. nudicaulis (L.) Hook.f., 218 App.

Lauraceae, 293 App., 387, 389, 392

Lavandula coronopifolia Poir., 201, 205-206, 220 App. pubescens Decne., 202, 205, 220 App.

Leandra aurea (Cham.) Cogn., 386 melastomoides Raddi, 386

Lectotypes

in Croton, 33, 36

in Desmos, 173-175*

in Gagea, 48 Fig.

in Lepidium, 165

in Pedicularis, 111-138*

in Xylopia, 339-341*

Leguminosae, 196 App., 221 App., 391, 406, 409

subfam. Caesalpinioideae, 348

subfam. Mimosoideae, 388-389

subfam. Papilionoideae, 389

Lentibulariaceae, 285
Leontodon

laciniatus (Bertol.) Widder, 218 App.

Leopoldia tenuiflorum (Tausch) Heldr., 207, 220 App.

Lepicochlea Rojas Acosta, 166 americana Rojas Acosta, 166

Lepidaploa tortuosa (L.) H.Rob., 291 App.

Lepidium L., 165-171* [166]

affghanum Boiss., 168

afghanicum (Rech.f. \& Köie) Al-Shehbaz \& Mummenhoff, 165, 168 appelianum Al-Shehbaz, 165-166 apterum (Lipsky) Al-Shehbaz \& Mummenhoff, 165, 168 aucheri Boiss., 216 App. botschantsevianum Al-Shehbaz, 168

botschantzevii (R.Vinograd.) Al-Shehbaz \& Mummenhoff, 165, 168 campestre (L.) W.T.Aiton, 167 chalepense L., 165, 167 coronopus (L.) Al-Shehbaz, 166 curvinervium (Botsch. \& Vved.)

Al-Shehbaz \& Mummenhoff, 165, 168 didymum L., 166-167

draba L., 166

flavum Torr., 167

gracile (Chodat \& Hassl.) Boelcke, 169

hindukushense Kitam., 170

latifolium L., 166

lipskyi (N.Busch) Al-Shehbaz \&

Mummenhoff, 165, 169

minutiflorum (Ridl.) Hewson, 167

niloticum (Delile) Sieber ex Steud., 166

olgae (R.Vinograd.) Al-Shehbaz \& Mummenhoff, 165, 169

orientale (Schrenk ex Fisch.) Al-Shehbaz \& Mummenhoff, 165, 167, 169

patrinoides (Regel) Al-Shehbaz \&

Mummenhoff, 165, 167, 169

pavlovii Al-Shehbaz \& Mummenhoff, 165, 169

perfoliatum L., 166

pterocarpum (Botsch. \& Vved.)

Al-Shehbaz \& Mummenhoff, 165, 169 sagittatum (Kar. \& Kir.) Al-Shehbaz, 167 sativum L., 166

silaifolium (Hook.f. \& Thomson)

Al-Shehbaz \& Mummenhoff, 165, 167, 170 
tiehmii (Rollins) Al-Shehbaz, 166

Lepisanthes, 1-9* mixta Leenh., 1-9 [3 Map, 7 Fig., 8]

Leptadenia pyrotechnica (Forssk.) Decne., 187, 194 App.

Lepus capensis arabicus Ehrenberg, 189, 208

Leucas inflata Benth., 187, 196 App.

Leysera leyseroides (Desf.) Maire, 206, 218 App.

Licania belemii Prance, 387

humilis Cham. \& Schltdl., 408

Lightfootia L'Hér., 366-367

Ligularia, 158 Rev.

Liliaceae, 43-59, 221 App.

Limeum arabicum Friedrich, 187-190, 196 App.

Limonium lobatum (L.f.) Chaz., 222 App.

Linaceae, 221 App.

Linaria simplex (Willd.) Desf., 222 App.

Linum, 221 App.

Lippia salviifolia Cham., 407

Lithocarpus Blume, 41

LLEWELlyn, O.A., 183-197, 199-224

Lloydia Salisb. ex Rchb., 43-44, 46-47 himalensis, 46 kunawurensis Royle, 44, 46-47, 54

Lobelia cardinalis L., 291 App.

comosa, 368 heyniana Roem. \& Schult., 308 jasminoides, 368

Lobeliaceae, 368

Loeflingia hispanica L., 216 App.

Lomelosia olivieri (Coult.) Greuter \& Burdet, 219 App. palaestina (L.) Raf., 219 App.

LOPES, S.F., 401-418

Lotononis platycarpa (Viv.) Pic.Serm., 221 App. Low, Y.W., 11-32

Loxogrammaceae, 329 Rev.
Ludwigia peruviana (L.) H.Hara, 282, 294 App.

Luehea paniculata Mart. \& Zucc., 389

Luffa, 425 cylindrica M.Roem., 428

Lycium shawii Roem. \& Schult., 197 App., 202, 206, 223 App.

Lycopodiaceae, 296 App.

Lycopodiella cernua (L.) Pic.Serm., 296 App.

Lycopodiophyta, 296 App.

Lythraceae, 221 App., 293 App.

Lythrum hyssopifolia L., 221 App.

Macaranga Thouars, 445, 456-457, 468

Machaerium acutifolium Vogel, 408 biovulatum Micheli, 278, 292 App. floribundum Benth., 292 App. isadelphum (E.Mey.) Amshoff, 278, 292 App. opacum Vogel, 408

Macrocarpaea (Griseb.) Gilg, 140

Macropeplus ligustrinus (Tul.) Perkins, 386, 391

Macrotyloma, 424 biflorum (Schumach. \& Thonn.) Hepper var. biflorum, 430

Madras Herbarium, 173-175*

Maerua crassifolia Forssk., 187, 194 App.

Maesobotrya Benth., 349

Magonia pubescens A.St.-Hil., 403, 407, 414

Malabayabas, 29

Malaysia, 450-451 Map, 453, 456

Malcolmia africana (L.) W.T.Aiton, 216 App.

Malesia, 443-482*

Mallotus Lour., 456-457 diadenus (Miq.) Müll.Arg., 464

Malpighiaceae, 293 App., 409

Malva parviflora L., 221 App.

Malvaceae, 221 App., 293 App., 331 Rev., 387-389, 392 


\section{Mandevilla}

hirsuta (Rich.) K.Schum., 278, 291 App. subsagittata (Ruiz \& Pav.) Woodson, 291 App.

Manudev, K.M., 257-263

Maprounea guianensis Aubl., 386, 407

Marantaceae, 331 Rev.

Marlierea clausseniana (O.Berg) Kiaersk., 386 laevigata (DC.) Kiaersk., 386

Matayba guianensis Aubl., 407

Matoniaceae, 329 Rev.

Matricaria aurea (Loefl.) Sch.Bip., 218 App.

Matthiola longipetala (Vent.) DC., 205, 216 App.

Maytenus, 281, 292 App. brasiliensis Mart., 387

dhofarensis Sebsebe, 306, 311-312

Medeiros, D., 33-38

Medicago laciniata (L.) Mill., 221 App. truncatula Gaertn., 221 App.

Medinilla, 158 Rev.

Melanolepis diadena Miq., 464

Melastomataceae, 274, 293 App., 387, 391, 409

Meliaceae, 29, 391

Meliosma, 158 Rev.

Mellivora capensis Schreber, 208

Menispermaceae, 196 App., 424, 431

Mentha longifolia (L.) L., 220 App.

Merciera A.DC., 351, 359, 364, 366-367, 369 azurea, 368 brevifolia A.DC., 354, 357, 361 Fig., 365, 368 eckloniana H.Buek, 354, 357, 368 leptoloba A.DC., 354, 357, 361 Fig., 368

Mesembryanthemum nodiflorum L., 214 App.

Mesotrophic cerradão, 401-418*

Miconia, 280 albicans (Sw.) Triana, 407 budlejoides Triana, 387 chamissois Naudin, 278, 282, 293 App. chartacea Triana, 386, 391

ciliata (Rich.) DC., 278, 281, 293 App.

dodecandra (Desr.) Cogn., 278, 293 App.

ferruginea (Desr.) DC., 407

ibaguensis (Bonpl.) Triana, 293 App.

impetiolaris (Sw.) D.Don ex DC., 294

App.

lacera (Bonpl.) Naudin, 294 App.

laevigata (L.) D.Don, 294 App.

rimalis Naudin, 386

theaezans (Bonpl.) Cogn., 386

Microcodon, 366, 369

glomeratus A.DC., 354, 357, 359, 361

Fig., 364, 366, 368

hispidulus (L.f.) Sond., 354, 356, 359-360

Fig., 363

sparsiflorus, 368

Micromeria imbricata (Forssk.) C.Chr., 220 App.

Micropholis gnaphaloclados (Mart.) Pierre, 386

Middleton, D.J., 328-330 Rev.

MilL, R.R., 61-109, 111-138, 159-163

Miller, A.G., 183-197, 199-224

Mimosa albida Humb. \& Bonpl. ex Willd., 292 App.

Minas Gerais, Brazil, 33-38*

Minuartia meyeri (Boiss.) Bornm., 209, 216 App.

Misopates orontium (L.) Raf., 222 App.

Mitreola petiolata (J.F.Gmel.) Torr. \& A.Gray, 306 Möller, M., 330-331 Rev.

Molluginaceae, 196 App.

Moltkiopsis ciliata (Forssk.) I.M.Johnst., 188, 194 App.

Monimiaceae, 157 Rev., 391

Monoploca Bunge, 166

Monsonia nivea (Decne.) Decne. ex Webb, 187, 195 App.

Monsoon, southwest, 301-319*

Montane grasslands, South Africa, 365

Moquinia racemosa (Spreng.) DC., 386

Moraceae, 221 App., 269-272, 387

Moraea sisyrinchium (L.) Ker-Gawl., 205, 209, 220 App. 


\section{Morettia}

canescens Boiss., 216 App.

parviflora Boiss., 194 App., 216 App.

Moricandia

sinaica (Boiss.) Boiss., 216 App.

Morinda

panamensis Seem., 295 App.

Moringa peregrina (Forssk.) Fiori, 187, 196 App.

Moringaceae, 157 Rev., 196 App.

Morphological phylogeny, 443-482*

Morphology, of Campanulaceae seed coats, 351-371

Moultonianthus, 450, 452-453 leembruggianus (Boerl. \& Koord.)

Steenis, 449-451

Mouriri exilis Gleason, 294 App.

Multivariate analysis, 401-418*

Mummenhoff, K., 165-171

Muscari tenuiflorum Tausch, 207, 209, 220 App.

Mussaenda leucophylla E.M.A.Petit, 434

soyauxii Büttner, 434

Mycorrhizae of Arum-type in Calolisianthus, 139-155* of Paris-type in Gentianaceae, 151

Myracrodruon urundeuva M.Allemão, 413

Myrceugenia alpigena (DC.) Landrum, 386

Myrcia, 406-407

amazonica DC., 386

anceps (Spreng.) O.Berg, 387

blanchetiana (O.Berg) Mattos, 386

guianensis (Aubl.) DC., 386

mutabilis (O.Berg) N.Silveira, 386

reticulosa Miq., 386

splendens (Sw.) DC., 294 App., 386, 391, 408

tomentosa (Aubl.) DC., 386, 391, 408

venulosa DC., 386

Myrciaria

floribunda (H.West ex Willd.) O.Berg, 386 glanduliflora (Kiaersk.) Mattos \& D.Legrand, 392

Myrica

cerifera L., 280-281, 294 App.

Myricaceae, 294 App., 329 Rev.
Myrsinaceae, 221 App., 294 App., 391

Myrsine, 408

coriacea (Sw.) R.Br. ex Roem. \& Schult., 278, 294 App.

guianensis (Aubl.) Kuntze, 386

umbellata Mart., 386, 391, 393, 409

venosa A.DC., 386

Myrtaceae, 29, 294 App., 387, 389, 391-392, 406, 409, 469

Namacodon Thulin, 353

NAMPY, S., 257-263

Narasimhan, D., 321-324

Nascimento, A.R.T., 401-418

Nasturtiolum Gray, 167

Nasturtiolum Medik., 167 castratum Medik., 167

National records, for Gabon, 423-442*

Nectandra gardneri Meisn., 389

leucantha Nees, 387

longicaudata (Lundell) C.K.Allen, 293

App.

nitida Mez, 293 App.

psammophila Nees, 387

salicifolia (Kunth) Nees, 293 App.

venulosa Meisn., 392

Neea

theifera Oerst., 408

Negripteris sciona (Chiov) Pic.Serm., 309

Nelumbo, 158 Rev.

Nelumbonaceae, 157 Rev., 329 Rev.

Neolepia W.A.Weber, 167 campestris (L.) W.A.Weber, 167

Neophron percnopterus L., 190, 208

Neotorularia torulosa (Desf.) Hedge \& J.Léonard, 216 App.

Nepal, 61-109*

Nerium, 158 Rev.

Neurada procumbens L., 188, 196 App., 206, 221

App.

Neuradaceae, 196 App., 221 App.

Neurolaena lobata (L.) R.Br. ex Cass., 291 App.

New combinations in Fagonia, 183-197 
in Lepidium, 165-171*

in Pedicularis, 61-109*

New Guinea, 1-9* [3 Map], 450-451 Map, $453,456,459$

New names

in Lepidium, 165-171*

in Xylopia, 339-341

New series

in Pedicularis, 61-109*, 111-138*

New species

in Appendicula, 321-324

in Begonia, 177-182*, 225-255*

in Diplycosia, 39-42*

in Eriocaulon, 257-263*

in Harpullia, 1-9

in Pedicularis, 61-109*

in Primula, 297-300*

in Rhinacanthus, 333-337

in Selliguea, 265-267*

in Thecacoris, 343-350*

in Vaccinium, 419-422*

New subspecies

in Omphalodes, 159-163*

New variety

in Pedicularis, 111-138*

Newman, M., 327-328 Rev.

Niangadouma, R., 423-442

Nicandra

physalodes (L.) Gaertn., 309, 313

Nitrariaceae, 221 App.

Noaea

mucronata (Forssk.) Asch. \& Schweinf., 206, 209, 215 App.

Noltie, H.J., 43-59

Nomenclature, of Gagea, 43-59

Nothofagus, 468

Notoceras

bicorne (Aiton) Amo, 202, 216

App.

Novitates Gabonenses 80, 423-442

Nyctaginaceae, 196 App., 222 App., 391

Ochnaceae, 294 App., 424, 431

Ochradenus

arabicus Chaudhary et al., 205, 209, 212, 223 App.

baccatus Delile, 197 App., 202, 205-206, 223 App.

Ocotea

beyrichii (Nees) Mez, 387 calliscypha L.C.S.Assis \& Mello-Silva, 392

lancifolia (Schott) Mez, 386

oppositifolia S.Yasuda, 386, 392

percoriacea (Meisn.) Kosterm., 386

pomaderroides (Meisn.) Mez, 386

spixiana (Nees) Mez, 386, 408

velloziana (Meisn.) Mez, 386

Olacaceae, 157 Rev., 387

Oldenlandia, 433

corymbosa L., 307

Olea

europaea

subsp. cuspidata (Wall. ex G.Don) Cif., 306, 311

Oleo-Juniperetalia procerae, 306, 315

Oligomeris

linifolia (Vahl) J.F.Macbr., 223 App.

Oman, 301-319* [303 Map, 310 Fig.]

Omphalea L., 444

Omphalodes, 159-163*

luciliae Boiss., 159-163

subsp. cilicica (Brand) Bornm., 160

Fig., 162

subsp. kurdica Rech.f. \& Riedl, 162

subsp. luciliae, 159, 162

subsp. pisidica R.R.Mill, 159-160 [160

Fig.], 162

subsp. scopulorum J.R.Edm., 161-162

[161 Fig.]

ripleyana P.H.Davis, 162

Onagraceae, 294 App.

Oncidium, 158 Rev.

Onobrychis

ptolemaica (Delile) DC., 221 App.

Ononis

sicula Guss., 221 App.

Orchidaceae, 294 App., 321-324*, 340

subfam. Epidendroideae, 321-324

tribe Podochileae, 321-324

Orobanchaceae, 61-109*, 111-138, 196

App., 222 App., 424, 431

Orobanche

aegyptiaca Pers., 222 App.

cernua Loefl., 222 App.

dhofarensis M.J.Y.Foley, 309, 312

ramosa, 222 App.

Oropetium

capense Stapf, 220 App.

Orthosiphon

pallidus Royle ex Benth., 307 


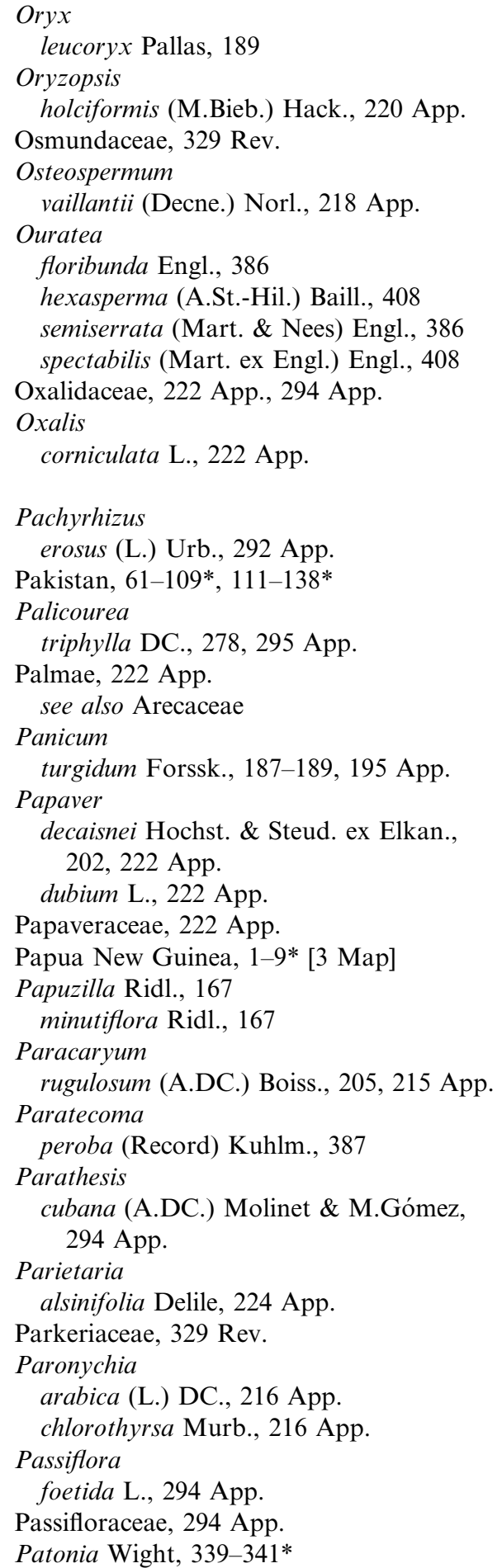

parvifolia Wight, 339-340

walkeri Wight, 339-340

Pattonia Wight, 340

Patzelt, A., 301-319

Pavetta longiflora Vahl, 307, 312

Pedaliaceae, 329 Rev.

Pediculares verticillatae Bunge, 62

Pedicularis L., 61-109*, 111-138

sect. Anodon Bunge, 114

sect. 'Brevirostres' Benth., 63, 112

sect. Cyclophyllum Bunge, 62

sect. 'Erostres' Benth., 63, 112

sect. 'Erostris' Benth., 112

sect. Lasioglossa H.L.Li, 114

sect. 'Longirostres' Benth., 63, 112

sect. Lophodon Bunge, 65

sect. Phanerantha H.L.Li, 111-138*

sect. Saccochilus T.Yamaz., 111-138*

sect. Schizocalyx H.L.Li, 112, 118, 125

sect. Siphonantha Bunge, 112, 117

sect. Siphonanthae Benth., 111-113,

117-118, 125

sect. Tubiflorae Bunge, 112

sect. Verticillatae Benth., 62-63

subsect. Rhinanthoides Hurus., 117

ser. Abrotanifoliae H.Limpr., 68, 124

ser. Axillares Maxim., 63

ser. Bicornutae R.R.Mill, 111, 124-125,

130

ser. Brevifoliae (Prain) Bonati, 61, 64-67, 71

ser. Comosae Maxim., 99

ser. Craspedotrichae Maxim., 124

ser. Debiles (Prain) Bonati, 65

ser. Dichotomae H.L.Li, 66-67

ser. Faucidentes, 112

ser. Flexuosae (Prain) Bonati, 65

ser. Franchetiana (Prain) Hurus., 114

ser. Franchetianae (Prain) H.L.Li, 114

ser. Garckeanae R.R.Mill, 118, 125

ser. Graciles Maxim., 63-64, 70-71

ser. Gyrorrhyncha (Prain) Hurus., 114

ser. Gyrorrhynchae (Prain) H.L.Li, 114

ser. Hirsutae Maxim., 65

ser. Ikomanae Hurus., 116

ser. Integrifoliae (Prain) H.L.Li, 64, 114

ser. Integrifoliae (Prain) Hurus., 114, 124

ser. Keiskeanae T.Yamaz., 116

ser. Kialenses H.L.Li, 117, 130 
ser. Klotzschianae R.R.Mill, 114-115,

118-119, 121, 124-125

ser. Longiflorae (Prain) Bonati, 114,

117-118, 124-125, 130

ser. Macranthae (Prain) Bonati, 114,

118-119

ser. Megalanthae (Prain) Bonati,

111-138* [130]

ser. Moupinenses P.C.Tsoong ex

H.B.Yang, 71

ser. Muscicolae Maxim., 112-114

ser. Myriophyllae Maxim., 64, 70-71

'ser.' Pectinatae Prain, 61-64

ser. Pectinatae (Prain) Bonati, 66, 68-71, 74

ser. Pectinatiformes P.C.Tsoong, 66 ser. Pectinatiformes P.C.Tsoong ex

H.B.Yang, 66-69, 72

ser. Pseudoerostres Hurus., 112

ser. Pseudomacranthae P.C.Tsoong ex

H.B.Yang, 121

ser. Pumiliones (Prain) Bonati, 114

ser. Rhinanthoides (Prain) Bonati, 113,

117-118

ser. Rhynchotrichae R.R.Mill, 61, 67, 70, 72

ser. Robustae (Prain) Hurus., 114, 118,

121, 125

ser. Semenowianae L.I.Ivanina \&

T.N.Popova, 116

ser. Semitortae (Prain) Bonati, 64, 66-68, 70

ser. Sikkimenses P.C.Tsoong ex

H.B.Yang, 66, 71

ser. Siphonanthae Benth., 112-114

ser. Sudeticae Maxim., 65

ser. Tantalorhynchae P.C.Tsoong ex

H.B.Yang, 71

ser. Tenuirostres Maxim., 61-109 [74]

ser. Tristes Benth., 114

ser. Verticillatae Benth., 62-63, 112

grex Rhizophyllum, 118

subgrex Rhizophyllum, 118

'tribe' Longirostres, 113

tribus Verticillatae Steven, 62

[unranked] Brevifoliae Prain, 64

[unranked] Brevitubae Prain, 114

[unranked] Capitatae Prain, 114

[unranked] Comosae Bunge, 65

[unranked] Debiles Prain, 65 [unranked] Eusiphonanthae Prain, 113

[unranked] Flexuosae Prain, 65

[unranked] Franchetianae [Franchetiana]

Prain, 114

[unranked] Gyrorrhynchae

[Gyrorrhyncha] Prain, 114

[unranked] Integrifoliae [Integrifolia]

Prain, 64, 114, 124

[unranked] Longiflorae Prain, 114

[unranked] Macranthae Bunge, 114

[unranked] Macranthae Prain, 114-115, 119,121

[unranked] Megalanthae Prain, 114, 116-117, 130

[unranked] Muscicolae Prain, 114

[unranked] Oppositifoliae Prain, 114

[unranked] Oxycarpae Prain, 117

[unranked] Pectinatae Bunge, 65, 74

$\alpha$ Elatae Bunge, 65

$\beta$ Humiles Bunge, 65

[unranked] Pectinatae Prain, 63-65, 72, 74

[unranked] Pumiliones Prain, 114

[unranked] Ramosae Bunge, 65

[unranked] Rhinanthoides Prain, 113

[unranked] Robustae Prain, 114, 117

[unranked] Semitortae Prain, 64-65

[unranked] Tenuirostres (Maxim.) Prain, 65

alopecuros Franch. ex Maxim., 64-66

anserantha T.Yamaz.

var. elevatogaleata (T.Yamaz.)

T.Yamaz., 124

armata Maxim., 113

atuntsiensis Bonati, 66-67, 71

bella Hook.f., 116

bicornuta Klotzsch, 111-138* [127]

var. adenocalyx R.R.Mill, 111, 126-129 [127, 128 Fig.]

var. bicornuta, 115 Fig., 127, 129

bipinnatifida (Pennell) R.R.Mill, 76, 82-83, 86, 89

birmanica Bonati, 61, 66-67, 70-71, 74

brevifolia D.Don, 63-64, 71, 132

caeruleoalbescens [caeruleo-albescens]

Wendelbo, 66-67, 75, 102

capitata Adams, 114

chinensis Maxim., 113

comosa L., 99

cornigera T.Yamaz., 117-121, 130-131 
cranolopha Maxim., 113

cristata Maxim., 64-65

cristata Vitmann, 64

cristatella Pennell \& H.L.Li, 64-65

cyrtorhyncha Pennell, 66-67, 76, 103

debilis Franch. ex Maxim., 65

densispica Franch. ex Maxim., 68

dichotoma Bonati, 66-67

duclouxii Bonati, 66-67

elata Willd., 65

elephantoides Benth., 111-138* [129]

elephas Boiss., 112-113

elwesii Hook.f., 114, 116, 121

eximia G.Watt, 111, 113, 123, 126-130

[128 Fig.]

fastigiata Franch., 117, 130

fetisowii Regel, 63-66

fletcheri P.C.Tsoong, 121

garckeana Prain ex Maxim., 118

gracilis Wall. ex Benth., 63, 132

gyrorhyncha Franch. ex Maxim., 64, 68

hoffmeisteri Klotzsch, 117-123, 130-133

[131, 133 Fig.]

hookeriana Wall. ex Benth., 111-113

ikomai Sasaki, 116

insignis Bonati, 119

instar Prain ex Maxim., 64

integrifolia Hook.f., 64

subsp. integerrima (Pennell \& H.L.Li)

P.C.Tsoong, 124

jainii Aswal \& Mehrotra, 61, 66-67, 74, 96, 99

kashmiriana Pennell, 66-67, 75, 99, 105

var. kashmiriana, 61

var. ornata Pennell, 61

keiskei Franch. \& Savat., 116

kialensis Franch., 117, 130

klotzschii Hurus., 113-115, 119

komarowii Bonati, 68

labellata Jacquem., 112-113

langsdorffii Fisch., 65

longiflora Rudolph, 73, 112-113, 120-121

var. tubiformis (Klotzsch) Pennell, 124

ludwigii Regel, 124

macrantha Klotzsch, 113-115, 119

macrantha Spreng., 114, 119

megalantha D.Don, 111-113, 116-124,

126, 130-132

var. hoffmeisteri (Klotzsch) Sealy, 132 var. pauciflora Prain ex Maxim., 117,

134

var. typica Prain, 131-132

f. hoffmeisteri (Klotzsch) Prain, 131-132

megalochila H.L.Li, 117-118, 121, 124,

130-131, 134

subsp. longituba T.Yamaz., 134

var. ligulata P.C.Tsoong, 131, 134

var. megalochila, 131, 134

f. megalochila, 131, 134

f. rhodantha, 131, 134

mollis Wall. ex Benth., 63

moupinensis Franch., 71

multiflora Pennell, 66-67, 75, 86, 96, 99, 101

murreeana R.R.Mill \& R.Bone, 69, 75, 83, 86, 92, 95-96 [95 Fig.]

muscicola Maxim., 113

ochroleuca Duthie ex Maxim., 115

ochroleuca J.C.Schloss., 115

oederi Vahl, 121

oliveriana Prain, 65

pauciflora (Prain ex Maxim.) Pennell,

117, 120-121, 123, 130-131, 134

pectinata Wall. ex Benth., 62-66, 69-71,

74, 76, 78-80, 82, 84, 86, 89, 92, 96

subsp. bipinnatifida Pennell, 80, 82-84,

86

subsp. palans (Prain) Pennell, 61, 78,

80, 82-84, 92, 95-96

subsp. palans sensu Yamazaki, 86, 89

subsp. pectinata, 78- 80

var. palans Prain, 80, 86, 92, 95-96

var. pyramidata (Royle ex Benth.)

Hook.f., 95-96

pectinatiformis Bonati, 66-68

perrottetii Benth., 112

porrecta Wall. ex Benth., 63, 65, 71

praealta Bonati, 66-67

przewalskii Maxim., 113

punctata Decne., 112-113

pyramidata Pallas ex Steven, 99

pyramidata Royle ex Benth., 61-66,

69-70, 74-75, 79-80, 86, 89, 96, 99,

103, 105, 121

subsp. kashmiriana (Pennell)

P.C.Tsoong, 99

subsp. multiflora (Pennell) P.C.Tsoong, 101 
subsp. pyramidata, 67

ramosissima Bonati, 66, 70-71

rhinanthoides Schrenk, 112, 116

rhynchotricha P.C.Tsoong, 61, 66-70, 72,

74

roborowskii Maxim., 63-65

scolopax Maxim., 63-65, 70-71

scullyana Prain ex Maxim., 113-114, 119,

124

semenowii Regel, 116

semitorta Maxim., 64-65, 70

siphonantha D.Don, 111-113, 116, 132

var. delavayi (Franch.) P.C.Tsoong, 124

smithiana Bonati, 66-67, 71

songarica Schrenk, 65

staintonii R.R.Mill, 69, 75, 80, 89, 91 Fig.

stewartii Pennell, 75, 85-86, 96

striata Pallas, 65

sudetica Willd., 65

tantalorhyncha Franch. ex Bonati, 71

tenuirostris Benth., 62-66, 69, 71, 74-76,

78, 96

tenuirostris sensu Aitchison, non Benth., 96, 99

tianschanica Rupr., 63-65

tristis L., 114

tsaii H.L.Li, 71

tubiflora Fisch., 112-113

verbenifolia Franch. ex Maxim., 65, 71

verticillata $\mathrm{L}$., 63

weixiensis H.P.Yang, 68

woodii R.R.Mill, 117, 120, 130-131, 134

yamazakiana R.R.Mill, 61, 69, 76, 82, 86,

89 Fig.

zhongdianensis H.B.Yang, 117, 120,

130-131

Peganum

harmala L., 221 App.

Pennisetum

divisum (J.F.Gmel.) Henrard, 195 App., 206, 220 App.

setaceum (Forssk.) Chiov, 205, 220 App.

Pentaphylacaceae, 294 App., 329 Rev.

Pera glabrata (Schott) Poepp. ex Baill., 386, 391

Pergularia

tomentosa L., 194 App., 215 App.

Periploca

aphylla Decne., 202, 205, 215 App.
Persea

splendens Meisn., 386

Peru, 177-182*

Petrorhagia cretica (L.) P.W.Ball \& Heywood, 206, 209, 212, 216 App.

Phagnalon schweinfurthii Sch.Bip. ex Schweinf., 218 App.

viridifolium Decne. ex Boiss., 201

var. omanense Qaiser \& Lack, 202-203, 207, 209, 218 App.

Phalaris minor Retz., 220 App.

Philippines, 11-32* [16, 24 Maps], 450-451 Map, 453, 456, 459

flora of, 39-42*

Phoenix dactylifera, 204-205 [204 Fig.], 222 App.

Photinia microcarpa Standl., 295 App.

Phyllanthaceae, 222 App., 325 Rev., $343-350^{*}$

Phyllanthus maderaspatensis L., 206, 222 App.

Phylogeny of Campanulaceae, 351-371* morphological, 443-482*

Physolepidium Schrenk, 167 repens Schrenk, 167

Phytogeography, 373-400*

of Belize, 273-296*

of Oman, 301-319*

Phytosociology, of Oman, 301-319*

Picris

babylonica Hand.-Mazz., 218 App. cyanocarpa Boiss., 202, 205, 218 App.

Pilocarpus giganteus Engl., 388

Pimelodendron, 469

Pimpinella schweinfurthii Asch., 307

Pinaceae, 295 App.

Pinophyta, 295 App.

Pinus, 274, 277, 279, 284 caribaea Morelet, 273-296* var. caribaea, 279-282 [280 Fig.], 285, 295 App.

var. hondurensis (Sénécl.)

W.H.G.Barrett \& Golfari, 274 
oocarpa Schiede ex Schltdl., 274, 276

var. ochoterenae Martínez, 274

var. oocarpa, 280 Fig.

tecunumanii, F.Schwerdtf. ex Eguiluz \&

Piper

J.P.Perry, 273-296* [280 Fig.]

aduncum L., 294 App.

cernuum Vell., 386

Piperaceae, 294 App.

Piptatherum

holciforme (M.Bieb.) Roem. \& Schult., 220 App.

Piptocarpha rotundifolia (Less.) Baker, 408

Pistachio-Eucleetalia schimperi, 307, 315

Pithecellobium lanceolatum (Humb. \& Bonpl. ex Willd.) Benth., 292 App.

Pittoniotis protracta (Bartl. ex DC.) Griseb., 281, 295 App.

Pittosporaceae, 329 Rev.

Pittosporum Banks ex Gaertn., 41

Plantaginaceae, 222 App., 294 App.

Plantago

afra L., 222 App.

amplexicaulis Cav., 222 App.

boissieri Hausskn. \& Bornm. ex Bornm., 222 App.

ciliata Desf., 222 App.

coronopus L., 222 App.

ovata Forssk., 222 App.

Plathymenia

reticulata Benth., 407

Plectranthus

barbatus Andrews, 308

Plenckia

populnea Reissek, 386, 408

Plinia

espinhacensis Sobral, 392

Plumbaginaceae, 222 App.

Poaceae, 195 App., 219 App., 294 App., 312,431

Podocarpaceae, 329-330 Rev.

Polyandrococos caudescens (Mart.) Barb.Rodr., 387

Polycarpaea repens (Forssk.) Asch. \& Schweinf., 194 App., 216 App. robbairea (Kuntze) Greuter \& Burdet, 216 App.

Polycarpon tetraphyllum (L.) L., 216 App.

Polygala, 205 abyssinica R.Br. ex Fresen., 222 App. erioptera DC., 187, 196 App. irregularis Boiss., 196 App. negevensis Danin, 222 App. schwartziana Paiva, 223 App. tinctoria Vahl, 309, 312

Polygalaceae, 196 App., 222 App., 312

Polygonaceae, 197 App., 223 App.

Polygonum argyrocoleon Steud. ex Kunze, 223 App.

Polypodiaceae, 265-267*, 329 Rev., 424, 432

Polypodiophyta, 296 App.

Polypodium cruciforme Ching, 265

Polypogon monspeliensis (L.) Desf., 220 App.

Pometia, 469

Portulaca oleracea L., 223 App.

Portulacaceae, 223 App., 329 Rev.

Posoqueria latifolia (Rudge) Roem. \& Schult., 386

Pouteria microstrigosa T.D.Penn., 388 ramiflora (Mart.) Radlk., 386 torta (Mart.) Radlk., 408

Pradeep, A.K., 257-263

Primula L., 297-300 sect. Chartacea Balf.f., 297

fenghwaiana C.M.Hu \& G.Hao, 297-298 [298 Fig.]

petelotii W.W.Sm., 297-299

Primulaceae, 297-300*

Prismatocarpus L'Hér., 359, 364, 366-367, 369

brevilobus, 368

campanuloides (L.f.) Sond., 354, 356, 368

crispus L'Hér., 354, 358-359, 362 Fig., 365-366, 368-369

diffusus (L.f.) A.DC., 354, 356, 360 Fig., 368

fruticosus L'Hér., 354, 356, 368

nitidus L'Hér., 354, 356, 368 
pedunculatus (P.J.Bergius) A.DC., 354,

356, 360 Fig., 368

schlechteri, 368

sessilis Eckl. ex A.DC., 354, 356,

363-364, 368

Procavia syriaca Hemprich \& Ehrenberg, 208

Proteaceae, 391-392

Protium

brasiliense (Spreng.) Engl., 386

heptaphyllum (Aubl.) Marchand, 386, 407

spruceanum (Benth.) Engl., 386

Prunus

myrtifolia (L.) Urb., 386

Pseudobombax

longiflorum (Mart. \& Zucc.) A.Robyns, 389

riopretense Ravenna, 392

Pseudocalyx aurantiacus Benoist, 426

Pseudosabicea, 436

sanguinosa, 424

Psidium, 408

guineense Sw., 386

rufum DC., 409

Psilotaceae, 329 Rev.

Psychotria, 424

biaristata Bartl. ex DC., 295 App.

costivenia Griseb., 295 App.

elata (Sw.) Hammel, 295 App.

poeppigiana Müll.Arg., 295 App.

vellosiana Benth., 386

Psydrax

arnoldiana (De Wild. \& T.Durand)

Bridson, 434

gilletii (De Wild.) Bridson, 434

Pteranthus

dichotomus Forssk., 216 App.

Pteridaceae, 223 App., 329 Rev.

Pteridium caudatum (L.) Maxon, 296 App.

Pterocephalus brevis Coult., 206, 209, 219 App.

Pterodon pubescens (Benth.) Benth., 407

Pterogaillonia calycoptera (Decne.) Lincz., 206, 223 App.

Pulicaria crispa (Forssk.) Benth. ex Oliv., 194 App., 202, 218 App. glutinosa (Boiss.) Jaub. \& Spach, 194 App.

incisa (Lam.) DC., 203, 218 App.

undulata (L.) C.A.Mey., 194 App., 202,

218 App.

vulgaris Gaertn., 218 App.

Quadrilátero Ferrífero, Brazil, 373-400*

[376 Map]

Qualea, 406

grandiflora Mart., 406-407, 414

parviflora Mart., 407

Quercus, 279, 281, 283, 286, 292 App.

oleoides Schltdl. \& Cham., 274

purulhana Trel., 278-279, 281, 283, 286, 292 App.

segoviensis Liebm., 292 App.

Radermachera Zoll. \& Moritzi, 41

Randia

cookii Standl., 278, 295 App.

speciosa Hook., non DC., 18

whitfordii (Elmer) Merr., 30

RATTER, J.A., 401-418

Redefinition, of Ficus trivia, 269-272

Rediscovery, of Croton josephinus, 33-38*

Refuge, in Saudi Arabia, 199-224*

Reichardia tingitana (L.) Roth, 218 App.

Relict species, in Saudi Arabia, 199-224*

Remijia obscura Blanco, 30

Remusatia vivipara (Roxb.) Schott, 308

Reseda muricata C.Presl, 197 App.

Resedaceae, 197 App., 223 App.

Revisions

of Endospermum, 443-482

of Gardenia, 11-32

of Pedicularis, 61-109, 111-138

Rhamnaceae, 197 App., 223 App., 388

Rhamnus staddo A.Rich., 306

Rhanterium epapposum Oliv., 194 App., 206, 218 App.

Rhazya stricta Decne., 188, 194 App., 215 App.

Rhigiophyllum Hochst., 352, 369 squarrosum Hochst., 354, 357, 359, 362

Fig., 365-366, 368 
Rhinacanthus Nees, 333-337*

dichotomus (Lindau) I.Darbysh.

var. emaculatus I.Darbysh., 336

flavovirens Amarasinghe \& Wijesundara, 333-337 [334-335 Figs]

nasutus (L.) Kurz, 333, 335-337 [335 Fig.]

polonnaruwensis Cramer, 333, 335-337

[335 Fig.]

rottlerianus Nees, 336

zeylanicus, 333

Rhus

somalensis Engl., 309

tripartita (Ucria) Grande, 202, 215 App.

Rhynchosia

minima (L.) DC.

var. minima, 306

pulverulenta Stocks, 196 App.

schimperi Hochst. ex Boiss., 196 App.

Rhysotoechia etmanii W.N.Takeuchi, 8

Richeria, 326 Rev.

grandis Vahl, 386

RodRigues, R.F., 401-418

Roella L., 359, 364, 366-367, 369

amplexicaulis Wolley-Dod, 354, 356, 368

arenaria Schltr., 354, 356, 368

ciliata L., 354, 356, 360 Fig., 368

cuspidata Adamson, 354, 356, 368

incurva A.DC., 354, 356, 368

muscosa L.f., 354, 356, 363, 368

prostrata, 368

psammophila, 368

secunda H.Buek, 354, 356, 360 Fig., 364, 368

squarrosa P.J.Bergius, 354, 356, 368

Roemeria

hybrida (L.) DC., 203, 222 App.

Rosaceae, 295 App.

Rostraria

cristata (L.) Tzvelev, 220 App. pumila (Desf.) Tzvelev, 220 App.

Rothmannia, 29-30

hispida (K.Schum.) Fagerl., 434

lateriflora (K.Schum.) Keay, 434

Rottlera

diadena (Miq.) Scheff., 464

Roupala

montana Aubl., 386, 391, 408

rhombifolia Mart. ex Meisn., 386
Rubiaceae, 11-32*, 152, 197 App., 223

App., 295 App., 312, 330 Rev.,

387-388, 409, 424, 432

subfam. Ixoroideae, 11

tribe Gardenieae, 11-32*

Rudgea

reticulata Benth., 387

Rumex

nervosus Vahl, 223 App.

vesicarius L., 202, 205, 223 App.

Rungia

pectinata Nees, 306

Russelia sarmentosa Jacq., 294 App.

Rutaceae, 197 App., 295 App., 388, 391

Ruttya fruticosa Lindau, 306

Rytigynia gossweileri Robyns, 435

laurentii (De Wild.) Robyns, 435

membranacea (Hiern) Robyns, 435

neglecta (Hiern) Robyns, 435

pauciflora (Schweinf. ex Hiern)

R.D.Good, 435

rubra Robyns, 435

senegalensis Blume, 435

umbellulata (Hiern) Robyns, 435

Sabicea, 436

apocynacea (K.Schum.) Razafim., 435

laxa Wernham, 436

longipetiolata De Wild., 436

sanguinosa (N.Hallé) Razafim., 436

schumanniana Büttner, 436

urceolata Hepper, 436

venosa Benth., 436

Salacia

crassifolia (Mart. ex Schult.) G.Don, 408

Salicaceae, 295 App., 325 Rev., 388

Salix, 97

Salsola, 193 App.

imbricata Forssk., 215 App.

spinescens, 193 App.

Salvertia convallariodora A.St.-Hil., 407

Salvia, 205

aegyptiaca L., 196 App., 220 App.

deserti Decne., 220 App.

lanigera Poir., 220 App.

spinosa L., 220 App. 
San Pastor Savanna, Belize, 273-296

[275 Map]

anthropogenic impacts on, 287

floristics of, 284

human use of, 284

physiognomy of, 285

soils of, 286

vegetation classification of, 279, 284

SANO, P.T., 373-400

Santalaceae, 223 App.

SAntos, M.F., 373-400

Sapindaceae, 1-9*, 295 App., 469

Sapindus

saponaria L., 295 App.

Sapium

glandulosum (L.) Morong, 386

Sapotaceae, 388

Saudi Arabia, 183-197*, 199-224*

Saudi Wildlife Commission (SWC), 183-197, 199-224

Sauvagesia erecta L., 278, 294 App.

Savanna in Belize, 273-296* [275 Map] tall grass, 301-319* [310 Fig.]

Savignya parviflora (Delile) Webb, 216 App.

Scabiosa olivieri Coult., 219 App. palaestina L., 219 App.

Scandix pecten-veneris L., 224 App.

Scanning electron microscopy, 351-371*

Schefflera

macrocarpa (Cham. \& Schltdl.) Frodin, 407

Schimpera

arabica Hochst. \& Steud., 216 App.

Schinziella, 425 tetragona (Schinz) Gilg, 430

Schippia concolor Burret, 278-279, 291 App.

Schismus arabicus Nees, 220 App. barbatus (L.) Thell., 220 App.

Schizaceae, 329 Rev.

Schizachyrium brevifolium (Sw.) Nees ex Büse, 309

Schizandraceae, 329 Rev.

Scirpoides holoschoenus (L.) Soják, 205, 219 App.

Scirpus vulgaris L., 219 App.

Scleria bracteata Cav., 278, 292 App.

Sclerocephalus, 194 App. arabicus Boiss., 194 App., 216 App.

Sclerolobium aureum (Tul.) Baill., 407 paniculatum Vogel, 401, 406-407, 409, 411,414

Scorzonera musilii Velen., 202, 218 App. papposa DC., 218 App. schweinfurthii Boiss., 218 App. syriaca Boiss. \& C.I.Blanche, 218 App. tortuosissima Boiss., 194 App.

Scorzoneroides laciniata (Bertol.) Greuter, 218 App.

Scrophularia arguta Aiton, 309

deserti Delile, 223 App. syriaca Benth., 202, 206, 223 App.

Scrophulariaceae, 61-109*, 111, 124, 127, 223 App., 312

Searsia tripartita (Ucria) Moffett, 202, 205-206, 209, 215 App.

Sebastiania longicuspis Standl., 292 App.

Sedum hispanicum L., 218 App.

Seed coat morphology, in Campanulaceae, 351-371

Seetzenia lanata (Willd.) Bullock, 197 App., 224 App.

Selaginellaceae, 329 Rev.

Selliguea, 265-267* cruciformis (Ching) Fraser-Jenk., 266-267 [267 Fig.]

pui Hovenkamp, 265-267 [267 Fig.]

Senebiera DC., 167

Senecio flavus (Decne.) Sch.Bip., 218 App. glaucus L., 205, 218 App.

Senefeldera verticillata (Vell.) Croizat, 388

Senna holosericea (Fresen.) Greuter, 196 App. 
italica Mill., 196 App., 203, 221 App. macranthera (Collad.) H.S.Irwin \&

Barneby, 386

obtusifolia (L.) H.S.Irwin \& Barneby, 307 peralteana (Kunth) H.S.Irwin \& Barneby, 292 App.

Senna-VAlle, L. De, 33-38

Senra

incana Cav., 309

SERAFIM, H., 373-400

Sericanthe auriculata (Keay) Robbr., 436

Serissa pinnata Blanco, 30

Serra de Caldas State Park, Brazil, 401-418 [404 Map]

Serra de São José, Brazil, 33-38

Setaria intermedia Roem. \& Schult., 308 pumila (Poir.) Roem. \& Schult., 305, 311-312

Sida

linifolia Cav., 278, 293 App.

ovata Forssk., 309

Silene

apetala Willd., 216 App. arabica Boiss., 216 App. burchellii Otth ex DC., 216 App.

linearis Decne., 217 App. schweinfurthii Rohrb., 217 App. villosa Forssk., 217 App.

SiLvA, L.C., 139-155

Simarouba amara Aubl., 386 versicolor A.St.-Hil., 407

Simmondsiaceae, 157 Rev.

Sino-Himalayan region, 111-138*

Sinopteridaceae, 329 Rev.

Siparuna guianensis Aubl., 386, 409

Siphocodon Turcz., 351-352, 366, 369 debilis Schltr., 354, 358, 362 Fig., 363, 365, 368

spartioides Turcz., 354, 358, 363-365 [364 Fig.], 368

Siphoneugena densiflora O.Berg, 386

Sisymbrium erysimoides Desf., 205, 216 App. irio L., 216 App. orientale L., 216 App.

Sisyrinchium

tinctorium Kunth, 293 App.

Smilacaceae, 295 App.

Smilax velutina Killip \& C.V.Morton, 295 App.

Solanaceae, 197 App., 223 App., 295 App.

Solanum

cladotrichum Dunal, 386

forskalii Dunal, 223 App.

incanum L., 307

jamaicense Mill., 282, 295 App.

nigrum L., 223 App.

villosum Forssk., 223 App.

Sonchus

oleraceus L., 218 App.

South Africa, 351-371

South Asia, 339-341

Species distribution patterns, in Brazil, 373-400

Spergula fallax (Lowe) E.H.L.Krause, 217 App.

Spergularia diandra (Guss.) Heldr., 217 App.

Spermacoce, 424 bequaertii (De Wild.) Verdc., 437 capitata Ruiz \& Pav., 278, 295 App. quadrisulcata (Bremek.) Verdc., 437 stachydea DC., 437

Spermatophyta, 291 App.

Sphenoclea zeylanica Gaertn., 352

Sphenocleaceae, 352

Sprengeria Greene, 167 flava (Torr.) Greene, 167

Sri Lanka, 333-337*, 339-341*

Stachytarpheta, 36 ajugaefolia Schauer, 36 sellowiana Schauer, 36

Stathmostelma, 425 welwitschii Britten \& Rendle, 426 var. welwitschii, 426

Sterculia curiosa (Vell.) Taroda, 388 macrophylla Vent., 478

Sterculiaceae, 348, 478

STÉvarT, T., 423-442

Stigmaphyllon ellipticum (Kunth) A.Juss., 293 App. lindenianum A.Juss., 293 App. 
Stipa, 195 App.

capensis Thunb., 220 App.

hohenackeriana Trin. \& Rupr., 195 App.

tortilis, 202

Stipagrostis, 189, 195 App.

ciliata (Desf.) De Winter, 202, 206, 220

App.

drarii (Tackh.) De Winter, 189, 195 App.

foëxiana (Maire \& Wilczek) De Winter, 195 App.

hirtigluma (Steud. ex Trin. \& Rupr.) De

Winter, 220 App.

obtusa (Delile) Nees, 195 App., 203, 220

App.

plumosa (L.) Munro ex T.Anderson, 206, 220 App.

Stipularia, 436

Strandveld, South Africa, 365

Strigosella

africana (L.) Botsch., 216 App.

Stroganowia Kar. \& Kir., 165-167

gracilis Pavlov, 169

sagittata Kar. \& Kir., 167

tiehmii Rollins, 166

Strongylodon, $158 \mathrm{Rev}$.

Stryphnodendron adstringens (Mart.) Coville, 389, 408 polyphyllum Mart., 407

Stubendorffia Schrenk ex Fisch., 165-171* afghanica Rech.f. \& Köie, 168 aptera Lipsky, 168, 170 botschantzevii R.Vinograd., 168 curvinervia Botsch. \& Vved., 168 gracilis (Pavlov) Botsch. \& Vved., 169 lipskyi N.Busch, 169

olgae R.Vinograd., 169

orientalis Schrenk ex Fisch., 167, 169

pterocarpa Botsch. \& Vved., 169

subdidyma N.Busch, 166

Stuppy, W., 325-327 Rev.

Styrax

camporum Pohl, 386, 406-407

ferrugineus Nees \& Mart., 407, 409

rotundatus (Perkins) P.W.Fritsch, 386

Sulawesi, Indonesia, 225-255* [227 Map]

Sulipa

pseudopsidium Blanco, 28-29

Sulitia, 30

obscurinervia (Merr.) Ridsdale, 30

Sumathi, R., 321-324
Sumatra, 419-422, 450-451 Map, 456

Swietenia macrophylla King, 287

Symbolanthus G.Don, 140, 153

Symplocaceae, 330 Rev.

Symplocos crenata (Vell.) Mattos, 386

nitens (Pohl) Benth., 386

Syngonanthus, 425

schlechteri Ruhland, 428

Synonymy, generic, 165-171*

Synopsis, of West African Thecacoris, 343-350

Systematics

of Calolisianthus, 139-155*

of Campanulaceae, 351-371

Syzygium Gaertn., 41

Tachigali Aubl., 411

TAKEUCHI, W., 1-9

Tamaricaceae, 224 App.

Tamarix

aphylla (L.) H.Karst., 224 App.

arabica Bunge, 224 App.

nilotica (Ehrenb.) Bunge, 224 App.

Tapirira

guianensis Aubl., 386, 391

obtusa (Benth.) J.D.Mitch., 386, 391

Tapirus

bairdii Gill, 287

Tapura

ivorensis Breteler, 348

Tarenna, 424

calliblepharis N.Hallé, 437

petitii N.Hallé, 437

Taxonomy

of Calolisianthus, 139-155

of Campanulaceae, 351-371*

of Endospermum, 443-482*

of Gagea, 43-59*

of Gardenia, 11-32*

Tayassu

pecari L., 287

tajacu Link, 287

Teвbitт, M.C., 177-182

Telanthophora, 278, 291 App.

Tephrosia, 196 App.

humilis Guill. \& Perr., 308

uniflora Pers., 188, 196 App.

Teramnus

repens (Taub.) Baker f. 
subsp. gracilis (Chiov) Verd., 306

Terminalia amazonia (J.F.Gmel.) Exell, 292 App. glabrescens Mart., 386

Ternstroemia carnosa Cambess., 386 tepezapote Schltdl. \& Cham., 294 App.

Tetraena, 197 App. simplex (L.) Beier \& Thulin, 197 App.

Tetrameristaceae, 330 Rev.

Tetrapogon villosus Desf., 205, 220 App.

Tetrastylidium grandifolium (Baill.) Sleumer, 387

Tetrorchidium Poepp., 444

Teucrium oliverianum Ging. ex Benth., 202, 206, 208 Fig., 220 App. polium L., 202, 205, 220 App. Thailand, 265-267*, 450-451 Map

Thecacoris A.Juss., 343-350* annobonae Pax \& K.Hoffm., 343, 345 cometia (Baill.) Leandri, 343

grandifolia (Pax \& K.Hoffm.) Govaerts, 343-345

humbertii Leandri, 343

leptobotrya (Müll.Arg.) Brenan, 344-345 lucida (Pax) Hutch., 343

madagascariensis A.Juss., 343-344

manniana (Müll.Arg.) Müll.Arg., 343, 348

membranacea Pax, 343, 348-349

micrantha Breteler, 343-348 [345, 346

Fig., 347 Map]

perrieri Leandri, 343

spathulifolia (Pax) Leandri, 343

stenopetala (Müll.Arg.) Müll.Arg., 343-345, 348-349

trichogyne Müll.Arg., 343, 345

viridis (Müll.Arg.) Leandri ex

G.L.Webster, 343-344, 349

Theilera E.Phillips, 359, 364, 366-367, 369 guthriei (L.Bolus) Phillips, 354, 356, 360

Fig., 364, 368

robusta (A.DC.) Cupido, 354, 356, 360

Fig., 364, 368

Themeda, 302-303, 310-315

quadrivalvis (L.) Kuntze, 301-319 [310

Fig.]

triandra Forssk., 220 App., 311, 313-315
Themedetalia-triandrae, 305, 314

Themedo-Hyparrhenietea, 301-319*

Thesium

humile Vahl, 223 App.

Thlaspi

campestre L., 167

Thомаs, D.C., 225-255

Thymelaea mesopotamica (C.Jeffrey) B.Peterson, 206, 209, 224 App.

Thymelaeaceae, 224 App.

Thyrsodium spruceanum Salzm. ex Benth., 388

Tibouchina candolleana (Mart. ex DC.) Cogn., 386 fissinervia (Schrank \& Mart. ex DC.)

Cogn., 386

Tiedt, L.R., 351-371

Torgos tracheliotus Forster, 191

Torilis leptophylla (L.) Rchb.f., 224 App.

Torricelliaceae, $330 \mathrm{Rev}$.

Tovomita leucantha (Schltdl.) Planch. \& Triana, 387

Tragus racemosus (L.) All., 195 App.

Treichelia Vatke, 351, 363, 365-366, 369 dodii Cupido, 354, 358, 362 Fig., 365, 368 longibracteata (H.Buek) Vatke, 355, 358, 362 Fig., 368

Trembleya parviflora (D.Don) Cogn., 386

Tribulus arabicus Hosni, 197 App. macropterus Boiss. var. arabicus (Hosni) F.Al-Hemaid \& Jacob Thomas, 187-190 [187 Fig.], 192, 197 App.

pentandrus Forssk., 197 App.

terrestris $\mathrm{L}$.

var. inermis Boiss., 205, 224 App. var. terrestris, 205, 224 App.

Tricalysia, 424 trachycarpa Robbr., 437

Trichodesma africanum (L.) Sm., 194 App., 215 App. ehrenbergii Schweinf. ex Boiss., 205, 215 App. 


\section{Trigonella}

glabra Thunb., 221 App.

hamosa L., 221 App.

stellata Forssk., 203, 205, 221 App.

Trigoniaceae, $330 \mathrm{Rev}$.

Trigynaea

oblongifolia Schltdl., 387

Trimezia, 36

Tripogon leptophyllus (A.Rich.) Cufod., 309

Tripsacum latifolium Hitchc., 282-283, 287, 294 App.

Trisetaria chaudharyana H.Scholz, 201, 203 Fig., 205, 209, 220 App.

Tristaniopsis, 29

Triumfetta pentandra A.Rich., 307

Triuridaceae, 157 Rev.

Trochodendraceae, 157 Rev.

Turkey, 159-163*

TURNER, I.M., 173-175, 339-341

Turnera aromatica Arbo, 295 App.

Turneraceae, 295 App., 330 Rev.

Tylophora parvifolia Robyns \& Lebrun, 426

Typification in Desmos, 173-175

in Gagea, 43-59

in Lepidium, 165-171*

Ulmaceae, 469

Umbelliferae, 224 App.

Umbilicus horizontalis (Guss.) DC., 218 App.

Unona, 173-175* viridiflora Bedd., 173-174 viridiflora Splitg. ex de Vriese, 174

Uranodactylus Gilli, 167 afghanicus Gilli, 167, 170 patrinoides (Regel) Gilli, 169 silaifolius (Hook.f. \& Thomson) Gilli, 170

Urbanodendron verrucosum (Nees) Mez, 387

Urticaceae, 224 App.

'Uruq Bani Ma'arid, Saudi Arabia, 183-197* [184 Map, 186 Fig.] checklist of plant taxa from, 193-197 App. conservation threats in, 191

interdune corridors of, 189

limestone plateau of, 187

qualification as an IPA, 190

sand dunes of, 188

socio-economics of, 191

Uvaria

brasiliensis Vell., 174

viridiflora Ruiz \& Pav. ex G.Don, 174

viridiflora (Splitg. ex de Vriese) Walp., 174

Vaccinium L., 419-422*

sect. Bracteata Nakai, 419-422

sect. Orianthe Schltr., 422

bartlettii Merr., 422

laurifolium (Blume) Miq., 422

lucidum (Blume) Miq., 419, 421-422

miquelii Boerl., 421

var. miquelii, 419, 421

myrtoides (Blume) Miq., 422

nagamasu Argent, 419-422 [420 Fig.]

scortechinii King \& Gamble, 419, 421

sumatranum Jack, 422

VALENTE, G.E., 139-155

Valerianaceae, 224 App.

Valerianella

oxyrhyncha Fisch. \& C.A.Mey., 206, 209, 224 App.

sclerocarpa Fisch. \& C.A.Mey., 206, 209, 224 App.

VAN Welzen, P.C., 443-482

Vangueriella, 424

laxiflora (K.Schum.) Verdc., 438

orthacantha (Mildbr.) Bridson \& Verdc., 438

Vantanea

compacta (Schnizl.) Cuatrec., 386

obovata (Nees \& Mart.) Benth., 386

Vatairea

macrocarpa (Benth.) Ducke, 407, 409

Vegetation types, 351-371*

Verbascum

sinaiticum Benth., 205, 223 App.

Verbenaceae, 36, 295 App.

Verbesina oerstediana Benth., 291 App.

Vernonanthura patens (Kunth) H.Rob., 291 App.

Vernonia cinerea (L.) Lees, 309 
Veronica

anagallis-aquatica L., 222 App.

anagalloides Guss., 222 App.

Vicia

monantha Retz., 221 App.

Vidalasia

morindifolia (Elmer) Tirveng., 30

Vietnam, 269-272*, 297-300*

Vigna, 424

pubigera Baker

var. pubigera, 430

radiata (L.) Wilczek, 307

stenophylla Harms, 430

Villaria

acutifolia (Elmer) Merr., 29

glomerata (Bartl. ex DC.) Mulyan. \&

Ridsdale, 30

littoralis Vidal, 29

odorata (Blanco) Merr., 29

philippinensis Rolfe, 29

Viola, 158 Rev.

cinerea Boiss.

var. stocksii (Boiss.) Becker, 307

Virola

sebifera Aubl., 408

Vismia

camparaguey Sprague \& L.Riley, 293 App.

Vitex

kuylenii Standl., 278-279, 293 App.

polygama Cham., 386

Voacanga

globosa (Blanco) Merr., 30

Vochysia, 406

cinnamomea Pohl, 409

emarginata Vahl, 386

haenkeana Pohl, 407

hondurensis Sprague, 295 App.

pyramidalis Mart., 386

rufa Mart., 408

thyrsoidea Pohl, 386

tucanorum Mart., 386, 391

Vochysiaceae, 295 App., 391, 406, 409, 414

Vulpes

rueppelli sabaea Pocock, 208

Vulpia

muralis (Kunth) Nees, 206, 209, 220 App.

Wahlenbergia Schrad. ex Roth, 351-371* acaulis E.Mey., 355, 358, 362 Fig., 363, 365-366, 368 adpressa (Thunb.) DC., 355, 357, 359,

361 Fig., 365, 368

androsacea, 368

annularis A.DC., 355, 357, 359, 361 Fig.,

365,368

axillaris, 368

campanuloides (Delile) Vatke, 216 App.

capensis (L.) A.DC., 355, 358, 363-365

[364 Fig.], 367-368

capillacea, 368

cernua (Thunb.) A.DC., 355, 357, 359,

365,368

cinerea, 368

cuspidata, 368

depressa, 368

desmantha, 368

ecklonii H.Buek, 355, 358, 362 Fig., 363, 365, 367-368

exilis A.DC., 355, 358, 362 Fig., 363, 365, 367-368

flexuosa (Hook.f. \& Thomson) Thulin, 306

fruticosa Brehmer, 355-356, 359-360

Fig., 364, 368-369

huttonii (Sond.) Thulin, 355, 357, 359,

361 Fig., 365, 368

juncea (H.Buek) Lammers, 355, 358,

363-365 [364 Fig.]

krebsii Cham., 355, 357, 359, 361 Fig., 365, 367-369

longifolia A.DC., 355, 358, 362 Fig., 363,

365, 367-368

neorigida Lammers, 355-356, 359-360

Fig., 364, 368-369

oxyphylla, 368

paniculata (Thunb.) A.DC., 355, 357,

359, 362 Fig., 365, 368

parvifolia (P.J.Bergius) Adamson, 355,

357, 359, 361 Fig., 364, 368

polyantha Lammers, 355, 357, 363-365

[364 Fig.], 368

procumbens (Thunb.) A.DC., 355, 357,

359, 361 Fig., 365, 368

psammophila Schltr., 355, 358, 363-365

[364 Fig.], 368

subulata (L'Hér.) Lammers, 355, 357 ,

359, 361 Fig., 364, 368

suffruticosa Cupido, 355, 358, 363-365

[364 Fig.], 368

tenella, 368 
tenerrima, 368

thunbergiana, 368

undulata, 368

virgata Engl., 355, 357, 359, 365, 368

WALters, G., 423-442

Wedelia

acapulcensis Kunth

var. parviceps (S.F.Blake) Strother, 278, 291 App.

West Africa, 343-350*

Western Ghats, 173-175*

Wetlands, in Belize, 273-296*

Whitfieldia, 424

brazzei (Baill.) C.B.Clarke, 425

Wight, Robert, 339-341*

WiJesundara, D.S.A., 333-337

WILKIN, P., 43-59

Winklera Regel, 165-171*

afghanica (Rech.f. \& Köie) Hedge, 168

patrinoides Regel, 167, 169

subsp. chitralica Jafri, 169

silaifolia (Hook.f. \& Thomson) Korsh., 170

Winkleria Rchb., 167

Winteraceae, 391

Withania

somnifera (L.) Dunal, 223 App.

Wong, K.M., 11-32

Woodfordia

uniflora (A.Rich.) Koehne, 308

Xaté, 273, 284

Xerophytes, geophytic in Begonia, 177-182

Xiphopteris

cultrata (Bory ex Willd.) Schelpe, 432

Xizang, China, 61-109*
Xylopia L., 339-341* [340]

aromatica (Lam.) Mart., 406-407, 411, 414

frutescens Aubl., 281, 291 App.

muricata L., 340

parvifolia Schltdl., 339-340

parvifolia (Wight) Hook.f. \& Thomson, 339-340

patoniae I.M.Turner, 339-340

Xylopicrum

parvifolium (Wight) Kuntze, 340

Xyridaceae, 285

Xysmalobium

heudelotianum Decne., 427

Yunnan, China, 297-300

Zanthoxylum

juniperinum Poepp., 295 App.

rhoifolium Lam., 386, 391, 393, 409

ZARREI, M., 43-59

Zilla

spinosa (L.) Prantl, 202-203, 205, 216

App.

Zingiberaceae, 424, 438

Ziziphus

joazeiro Mart., 388

nummularia (Burm.f.) Wight \& Arn., 205, 223 App.

spina-christi (L.) Desf., 187, 197 App., 223 App.

Zoegea

purpurea Fresen., 205, 218 App.

Zygophyllaceae, 197 App., 224 App., 438

Zygophyllum

hamiense, 197 App. 